\title{
Active Suspension Control Based on Estimated Road Class for Off-Road Vehicle
}

\author{
Mingde Gong $\mathbb{D},{ }^{1,2}$ Haohao Wang, ${ }^{3}$ and Xin Wang ${ }^{1}$ \\ ${ }^{1}$ School of Mechanical Engineering, Yanshan University, Qinhuangdao 066004, China \\ ${ }^{2}$ Hebei Key Laboratory of Special Delivery Equipment, Yanshan University, Qinhuangdao 066004, China \\ ${ }^{3}$ School of Mechanical Science and Engineering, Jilin University, Changchun 130025, China \\ Correspondence should be addressed to Mingde Gong; 307070001@qq.com
}

Received 24 January 2019; Revised 30 May 2019; Accepted 16 June 2019; Published 27 June 2019

Academic Editor: Andrzej Swierniak

Copyright (c) 2019 Mingde Gong et al. This is an open access article distributed under the Creative Commons Attribution License, which permits unrestricted use, distribution, and reproduction in any medium, provided the original work is properly cited.

Road input can be provided for a vehicle in advance by using an optical sensor to preview the front terrain and suspension parameters can be adjusted before a corresponding moment to keep the body as smooth as possible and thus improve ride comfort and handling stability. However, few studies have described this phenomenon in detail. In this study, a LiDAR coupled with global positioning and inertial navigation systems was used to obtain the digital terrain in front of a vehicle in the form of a 3D point cloud, which was processed by a statistical filter in the Point Cloud Library for the acquisition of accurate data. Next, the inverse distance weighting interpolation method and fractal interpolation were adopted to extract the road height profile from the 3D point cloud and improve its accuracy. The roughness grade of the road height profile was utilised as the input of active suspension. Then, the active suspension, which was based on an LQG controller, used the analytic hierarchy process method to select proper weight coefficients of performance indicators according to the previously calculated road grade. Finally, the road experiment verified that reasonable selection of active suspension's LQG controller weightings based on estimated road profile and road class through fractal interpolation can improve the ride comfort and handling stability of the vehicle more than passive suspension did.

\section{Introduction}

In the running course of a vehicle, the road excitation characteristics considerably influence its ride comfort and handling stability $[1,2]$. Suspension is an important component that plays a critical function in the transmission of force between vehicle's tire and body and alleviates the impact of the road surface. Consequently, obtaining the pavement roughness in front of a vehicle to control its suspension and improve ride comfort and handling stability has been attracting the attention of researchers.

Existing active suspension regulates its parameters only when the road interferes with the vehicle $[1,2]$. The use of the sensor system to detect the road on which a vehicle will be running and of the built-in controllers to process the related data can maximise the potential of active suspension. Therefore, a suspension system with a preview function is vital to future intelligent chassis.
Stereo cameras [3,4] and LiDAR sensors [5-7] can be adopted for this function by measuring the road height information in front of a running vehicle with certain accuracy. In [2], the use of a low-cost ultrasonic sensor was examined to compute road surface height estimation in real time by using simple algorithms due to the drawbacks of sensors (i.e., stereo vision or LiDAR), such as costliness and computation complexity. However, the ultrasonic sensor does not have the high precision of a laser radar. In [7], a LiDAR coupled with a global positioning system (GPS) and an inertial navigation system (INS) was used to measure two off-road surfaces, but details of the measurement and data processing were not provided. In [8], the author proposed the use of the overall response of the preceding vehicles to generate preview controller information for follower vehicles; no sensor is used to measure the terrain online.

Although many scholars have proposed the adoption of preview information for active suspension, detailed 


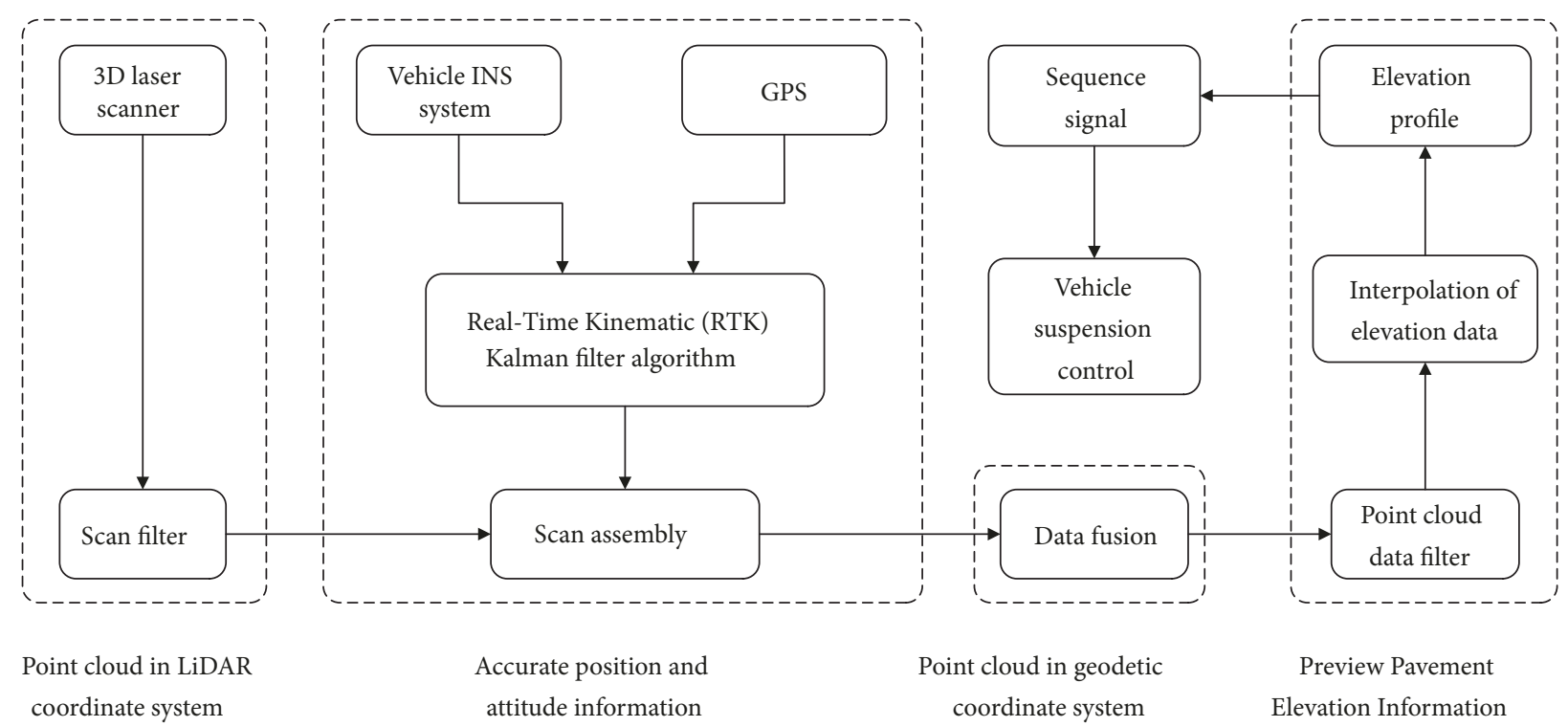

FIGURE 1: Mobile mapping system.

descriptions have not been provided. Instead, researchers have merely introduced concepts or assumed the road height profile to be known and obtained by statistical methods. This study proposes an approach that uses LiDAR coupled with INS and GPS to obtain a preview of the $3 \mathrm{D}$ point cloud of the terrain in a geodetic coordinate system and extract the road height profile in front of a vehicle. This work also elaborates the methods and steps of data processing. The road roughness grade is identified on the basis of the aforementioned information. Then, the LinearQuadratic-Gaussian (LQG) controller's weight coefficients of performance indicators are optimised in different road roughness grades. Finally, we select proper weight coefficients that correspond to previously calculated road grades to improve the vehicle's ride comfort and handling stability.

This study is organised as follows. Section 2 provides the mobile mapping system. Section 3 presents the data processing of sensors. Section 4 uses the methods of the two preceding sections to experiment and obtain the $3 \mathrm{D}$ point cloud of the test site. Section 5 adopts the road height profile information utilised in fractal interpolation theory in Section 6 to improve accuracy. This profile information is extracted from the $3 \mathrm{D}$ point cloud by using the inverse distance weighting (IDW) interpolation method. Section 7 solves the grade of the road height profile's roughness. Section 8 establishes the suspension vertical dynamic model and designs the LQG controller for active suspension. Section 9 calculates the controller's weight coefficients of performance indicators for different road grades using the analytic hierarchy process (AHP). In Section 10, a road experiment verifies that reasonable selection of LQG controller weightings based on estimated road profile and road class through fractal interpolation can improve the ride comfort and handling stability of the vehicle more than passive suspension does.

\section{Mobile Mapping System}

To control active suspension with preview information, a device that maps the front terrain of the running vehicle must be developed. Laser radar scanning is a rapid $3 \mathrm{D}$ measurement method and has existed for a while. But it is seldom used for the realization of active suspension. The mobile LiDAR system is mainly composed of a LiDAR sensor, GPS, and INS (Figure 1). In the running course, the LiDAR is mounted in front of the vehicle and returns the $3 \mathrm{D}$ point cloud of the road on the basis of its own coordinate system. One vital challenge in accurate mapping is estimating the attitude of LiDAR, which affects the precision of the point cloud. To solve this problem, the INS, which is installed near the LiDAR, collects the attitude angle that is used to compensate for motion during scan acquisition to improve the precision of data in real time. We need to realise the accurate location of the LiDAR to spread the 3D point cloud on the earth surface. Although GPS has higher positioning accuracy than other approaches, it is vulnerable to a wide variety of interferences, such as the multipath effect from radars, electromagnetic interference, and block of signals $[9,10]$. INS is a self-contained system that consists of inertial measurement units, which can provide dynamic measurement for a short period with high frequent update. It is a complete autonomous navigation system with remarkable concealment, strong anti-interference capability, and immunity to meteorological conditions [11]. However, its measurement error may accumulate over time due to drifting effects [12]. The integration of GPS and INS has been proposed and widely implemented for vehicle applications to leverage the strengths of the two systems and to offset their individual drawbacks because they are more advantageous than single navigation systems $[13,14]$. In these integrated systems, the Kalman filter is a popular fusion method that has been used in recent years due to its practicability and suitability [15]. The centimeter-level positioning accuracy can 


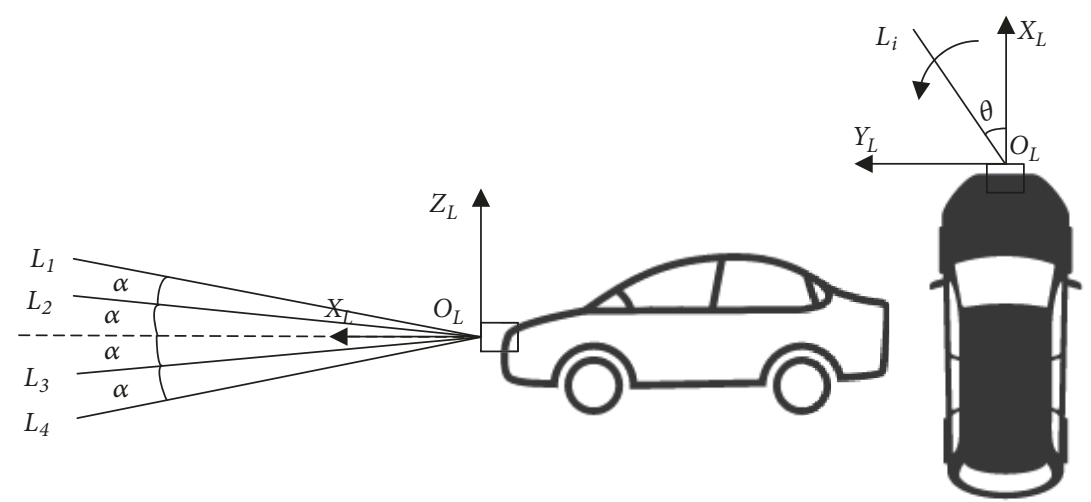

FIGURE 2: Operation mode of ibeo LUX 2010.

be obtained in real time by using real-time kinematic (RTK) technology.

\section{Point Cloud Data Processing}

The multilinear LiDAR can launch more one laser lines whilst rotating around the laser emission centre at a certain frequency and returns the point cloud of the obstacle in the form of relative distance $L_{i}$ and angle $\theta$, which depend on the LiDAR's coordinate system. The point cloud is stacked in front of the vehicle instead of being spread on the earth surface because the LiDAR coordinate system moves in real time with the vehicle. To resolve this problem, in the first step, we should coordinate the transformation of the point cloud. Relative distance $L_{i}$ and angle $\theta$ of the point cloud are transformed into the Cartesian coordinate. In the second step, data fusion algorithm is then implemented. GPS/INS integrated navigation system is used to transform the data obtained from first step into the WGS-84 geodetic coordinate system, which is stationary.

3.1. Coordinate Transformation of Point Cloud. The LiDAR's vertical angle resolution is marked as $\alpha$. Figure 2 shows that $O_{L}-X_{L} Y_{L} Z_{L}$ is a LiDAR coordinate system whose origin $O_{L}$ coincides with the laser emission centre. The angle between the instantaneous laser line and the $X_{L}$ axis is defined as $\theta$, and $L_{i}$ is the distance between $O_{L}$ and the obstacle. According to geometric relations, the $3 \mathrm{D}$ point cloud is represented in the Cartesian coordinate system as follows:

$$
\begin{gathered}
{\left[\begin{array}{l}
x_{L} \\
y_{L} \\
z_{L}
\end{array}\right]=L_{i} \cdot\left[\begin{array}{c}
\cos \alpha_{i}^{*} \cdot \cos \theta \\
\cos \alpha_{i}^{*} \cdot \sin \theta \\
\sin \alpha_{i}^{*}
\end{array}\right]} \\
\alpha_{i}^{*}= \begin{cases}2 \alpha, & i=1 \\
\alpha, & i=2 \\
-\alpha, & i=3 \\
-2 \alpha, & i=4\end{cases}
\end{gathered}
$$

3.2. Data Fusion. The coordinate systems of the mobile mapping system include the LiDAR coordinate system $O_{L}-$ $X_{L} Y_{L} Z_{L}$, INS coordinate system $O_{I}-X_{I} Y_{I} Z_{I}$, local horizontal coordinate system $O_{G}-X_{G} Y_{G} Z_{G}$, and WGS-84 geodetic coordinate system $O_{84}-X_{84} Y_{84} Z_{84}$ (Figure 3).

The coordinate values of the point cloud $\left[x_{L}, y_{L}, z_{L}\right]$ based on the LiDAR coordinate system are transformed to the WGS-84 geodetic coordinate system as follows:

$$
\begin{aligned}
& {\left[\begin{array}{l}
x_{W 84} \\
y_{W 84} \\
z_{W 84}
\end{array}\right]} \\
& =\left[\begin{array}{l}
x_{G P S} \\
y_{G P S} \\
z_{G P S}
\end{array}\right] \\
& +R_{W}\left[R_{N}\left[R_{M}\left[\begin{array}{l}
x_{L} \\
y_{L} \\
z_{L}
\end{array}\right]+\left[\begin{array}{l}
\Delta x_{L I} \\
\Delta y_{L I} \\
\Delta z_{L I}
\end{array}\right]-\left[\begin{array}{l}
\Delta x_{I G} \\
\Delta y_{I G} \\
\Delta z_{I G}
\end{array}\right]\right]\right]
\end{aligned}
$$

where $R_{M}$ is the rotation matrix between LiDAR and INS, whose element is calculated from the installation angle error of two sensors. The error angles around $x, y$, and $z$ are $\beta, \alpha$, and $\gamma$, which represent roll, pitch, and course angle errors, respectively, during the installation process.

$$
\begin{aligned}
& R_{M}=R_{\gamma z} \cdot R_{\alpha y} \cdot R_{\beta x} \\
& R_{\gamma z}=\left[\begin{array}{ccc}
\cos \gamma & \sin \gamma & 0 \\
-\sin \gamma & \cos \gamma & 0 \\
0 & 0 & 1
\end{array}\right] \\
& R_{\alpha y}=\left[\begin{array}{ccc}
\cos \alpha & 0 & -\sin \alpha \\
0 & 1 & 0 \\
\sin \alpha & 0 & \cos \alpha
\end{array}\right] \\
& R_{\beta x}=\left[\begin{array}{ccc}
1 & 0 & 0 \\
0 & \cos \beta & \sin \beta \\
0 & -\sin \beta & \cos \beta
\end{array}\right]
\end{aligned}
$$




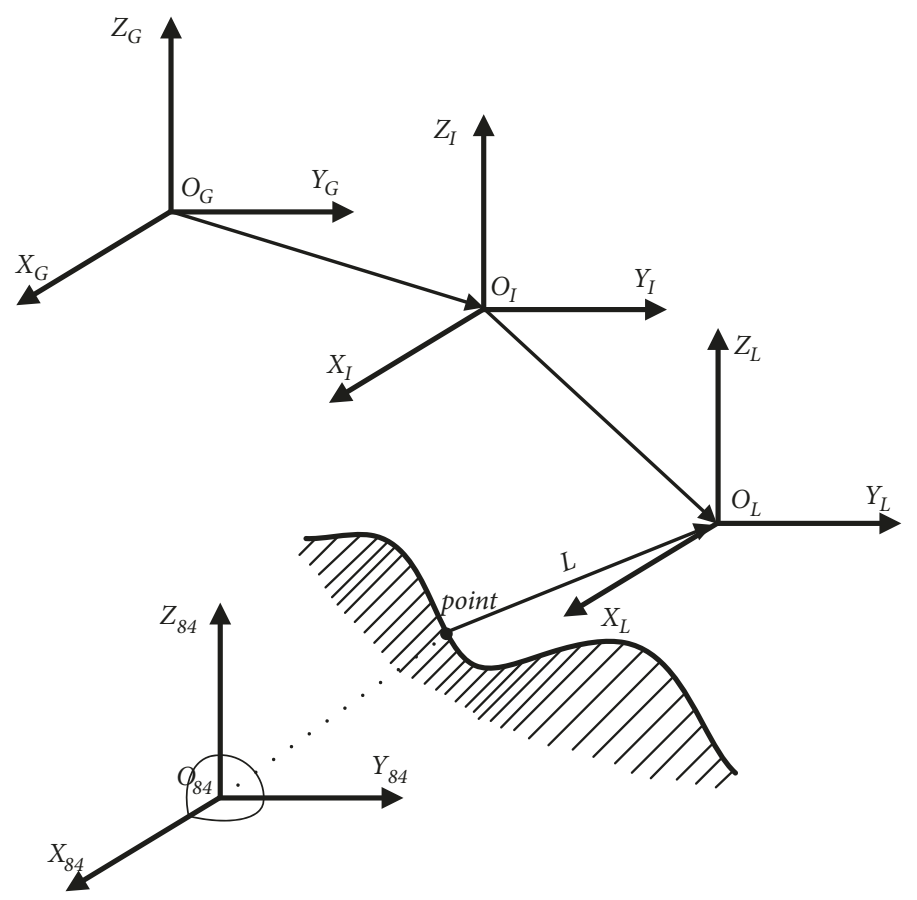

FIGURE 3: Coordinate systems of mobile mapping system.

where $R_{N}$ is the rotation matrix between INS and the local horizontal coordinate system and the matrix element is calculated from the measured values of INS, where $\varphi, \omega$, and $k$ represent pitch, roll, and course angles, respectively.

$$
\begin{aligned}
& R_{N}=R_{\kappa} \cdot R_{\varphi} \cdot R_{\omega} \\
& R_{\kappa}=\left[\begin{array}{ccc}
\cos \kappa & \sin \kappa & 0 \\
-\sin \kappa & \cos \kappa & 0 \\
0 & 0 & 1
\end{array}\right] \\
& R_{\varphi}=\left[\begin{array}{ccc}
\cos \varphi & 0 & -\sin \varphi \\
0 & 1 & 0 \\
\sin \varphi & 0 & \cos \varphi
\end{array}\right] \\
& R_{\omega}=\left[\begin{array}{ccc}
1 & 0 & 0 \\
0 & \cos \omega & \sin \omega \\
0 & -\sin \omega & \cos \omega
\end{array}\right]
\end{aligned}
$$

where $R_{W}$ is the rotation matrix between the local horizontal coordinate system and the WGS-84 geodetic coordinate system and the matrix element is calculated from the measured values of the loosely coupled GPS/INS system, where $B, L$, and $H$ represent latitude, longitude, and altitude, respectively.

$$
R_{W}=\left[\begin{array}{ccc}
-\cos L \sin B & -\sin L & -\cos L \cos B \\
-\sin L \sin B & \cos L & -\sin L \cos B \\
\cos B & 0 & -\sin B
\end{array}\right]
$$

where $\left[\Delta x_{L I}, \Delta y_{L I}, \Delta z_{L I}\right]^{T}$ are the offset values between the LiDAR and the INS coordinate system. $\left[\Delta x_{I G}, \Delta y_{I G}, \Delta z_{I G}\right]^{T}$ represent the offset values between the INS and the GPS coordinate system.

The location of GPS in the WGS-84 geodetic coordinate system can be expressed as follows:

$$
\left[\begin{array}{l}
x_{G P S} \\
y_{G P S} \\
z_{G P S}
\end{array}\right]=\left[\begin{array}{c}
\left(\frac{a}{\sqrt{1-e^{2} \sin ^{2} B}}+H\right) \cdot \cos B \cdot \cos L \\
\left(\frac{a}{\sqrt{1-e^{2} \sin ^{2} B}}+H\right) \cdot \cos B \cdot \sin L \\
{\left[\frac{a}{\sqrt{1-e^{2} \sin ^{2} B}} \cdot\left(1-e^{2}\right)+H\right] \cdot \sin B}
\end{array}\right]
$$

where $\alpha$ is a semimajor axis of WGS-84 and $e$ is the first eccentricity.

$$
\begin{aligned}
a & =6378137 m \\
e^{2} & =0.006694379
\end{aligned}
$$

\section{Terrain Measurement}

In this study, integrated navigation system of MEMS is adopted, of which core components are the gyroscope, accelerometer, and the high-performance Beidou/GPS receiver. The position, velocity, azimuth angle, attitude angle, three-axis acceleration, and angular velocity can be output by the Kalman filter integrated navigation algorithm that can solve the problem of INS error drift over time and RTK technology. It enters the pure inertial navigation mode without satellite navigation signal and can maintain high navigation accuracy in a short time.

The ibeo LUX $2010^{\circledR}$ selected for project implementation detects objects and their distance by means of laser beams. 
TABLE 1: Specification of integrated navigation system.

\begin{tabular}{lccc}
\hline $\begin{array}{l}\text { Azimuth } \\
\text { accuracy }\end{array}$ & $\begin{array}{c}\text { Attitude } \\
\text { accuracy }\end{array}$ & $\begin{array}{c}\text { Positioning } \\
\text { accuracy }\end{array}$ & $\begin{array}{c}\text { Speed } \\
\text { accuracy }\end{array}$ \\
\hline$\leq 0.1^{\circ}$ & $\leq 0.1^{\circ}$ & $\leq 2 \mathrm{~cm}$ & $\leq 0.1 \mathrm{~m} / \mathrm{s}$ \\
\hline Interface & Data update rate & Voltage & Power waste \\
\hline$R S 232$ & $\leq 500 \mathrm{~Hz}$ & $D C: 9 \mathrm{~V} \sim 36 \mathrm{~V}$ & $\leq 7 \mathrm{~W}$ \\
\hline
\end{tabular}

TABLE 2: Specification of LiDAR.

\begin{tabular}{lccc}
\hline $\begin{array}{l}\text { Scanning } \\
\text { frequency }\end{array}$ & $\begin{array}{c}\text { Angular } \\
\text { resolution }\end{array}$ & $\begin{array}{c}\text { Number of laser } \\
\text { lines }\end{array}$ & $\begin{array}{c}\text { Laser } \\
\text { wavelength }\end{array}$ \\
\hline $50 \mathrm{~Hz}$ & $0.5^{\circ}$ & 4 & $905 \mathrm{~nm}$ \\
\hline Laser grade & $\begin{array}{c}\text { Vertical } \\
\text { angular } \\
\text { resolution }\end{array}$ & Voltage & Interface \\
\hline Class I & $\alpha=0.8^{\circ}$ & DC: $: 9 \mathrm{~V} \sim 27 \mathrm{~V}$ & CAN/Ethernet \\
\hline
\end{tabular}

It scans the surroundings with several rotating laser beams, receives the echoes with a photo diode receiver, processes the data by means of a time of flight calculation, and issues the processed data via the interfaces Ethernet and/or CAN.

The specifications of integrated navigation system and LiDAR are shown in Tables 1 and 2.

The calibration of the integrated navigation system and the LiDAR needs to be carried out. The main work is to measure the relative position and angular error between the sensors.

The sensor position center is given by the manufacturer, so the relative position error between sensors is measured by a ruler, a triangle ruler, and the like.

Sensors are mounted on an adjustable platform. Integrated navigation system's pitch angle and roll angle are adjusted to 0 according to its output value, and the heading angle is made to coincide with the longitudinal symmetry axis of the vehicle by using a laser scale. Based on the adjusted integrated navigation system, the relative angle error between sensors is measured by a level meter.

The data rate of LiDAR is $50 \mathrm{~Hz}$ and that of integrated navigation system is $500 \mathrm{~Hz}$. In the process of data solution, a unified time stamp and Kalman filter prediction model are used to solve the problem of inconsistent working frequency of multisensor. The vehicle computer time is regarded as the unified time stamp of the system. According to Kalman filter prediction model, the sensor data are converted to the working frequency of the LiDAR, thus forming the multisensor data at the same frequency.

The dirt terrain around a university was selected as the test site (Figure 4). Multithreading technology was used to ensure simultaneous data acquisition from integrated navigation system and LiDAR. The contact surface between the tire and the ground is the reference zero surface. Figure 5 shows the road map of the selected dirt terrain after data processing in MATLAB ${ }^{\circledR}$.

Table 3 shows the point cloud properties of the dirt terrain.

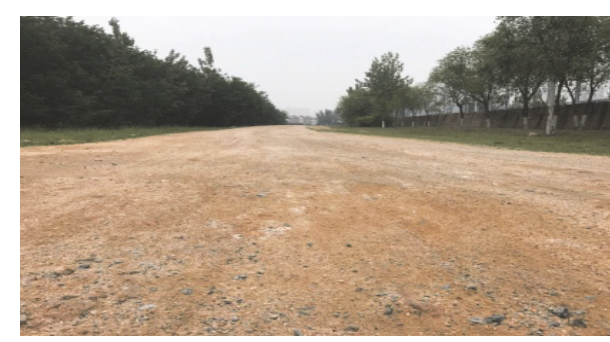

FIgURE 4: Test site.

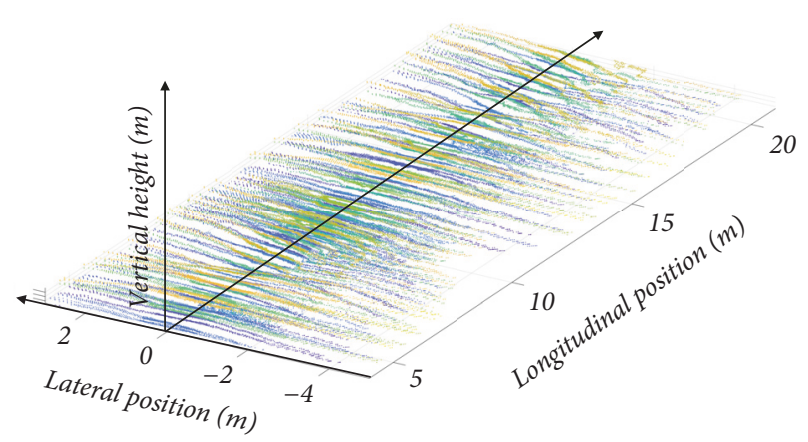

Figure 5: A road map of dirt terrain.

\section{Extraction of Road Height Profile}

The 3D point cloud of the dirt terrain acquired with LiDAR inevitably suffers from noise contamination and contains outliers [16-18] due to the limitations of sensors [19], the inherent noise of the acquisition device [20], and the lighting or reflective nature of the surface or artefact in the scene [21].

Therefore, the raw point cloud should be filtered to obtain accurate data that are suitable for further processing. For this project, we only consider the road that the vehicle is going to pass through and other road elevation information is ignored to simplify the calculation. Thus, the use of the road height profile extracted from the 3D point cloud of the terrain is beneficial. This paper assumes that the road ahead is empty. In the follow-up study, vehicle trajectory planning should be carried out in a short time according to the steering wheel angle.

5.1. Filtering of Point Cloud. The Point Cloud Library (PCL) is a large-scale open project [22] for $2 \mathrm{D} / 3 \mathrm{D}$ image and point cloud processing, the framework of which contains numerous state-of-the-art algorithms, including filtering, feature estimation, surface reconstruction, registration, model fitting, and segmentation. For example, these algorithms can be used to filter outliers from noisy data, stitch 3D point clouds together, segment relevant parts of a scene, extract key points, and compute descriptors to recognise objects in the world on the basis of their geometric appearance and create and visualise surfaces from point clouds.

The StatisticalOutlierRemoval filter in PCL is used to filter outliers. Compared with the unused filter, the denoised 3D point cloud of the dirt terrain decreased from 346036 to 322628 (Figure 6). 
TABLE 3: 3D point cloud properties of dirt terrain.

\begin{tabular}{lccc}
\hline Point numbers & \multicolumn{2}{c}{346036} & \\
\hline Longitudinal position range & & $4.0002(\mathrm{~m})$ & $20.9994(\mathrm{~m})$ \\
Lateral position range & Minimum & $-4.9935(\mathrm{~m})$ & Maximum \\
Vertical height range & & $-0.1477(\mathrm{~m})$ & $0.1999(\mathrm{~m})$ \\
\hline
\end{tabular}

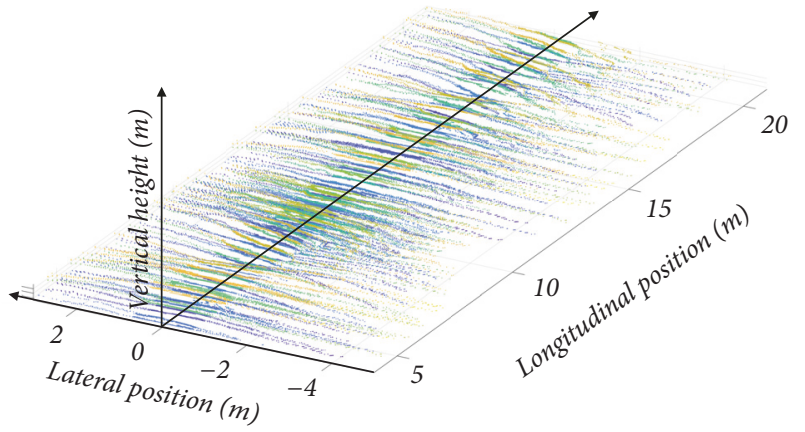

FIGURE 6: Denoised road map of the dirt terrain.

5.2. Extraction of Road Height Profile. We can predict the trajectory of a vehicle in advance according to the vehicle's driving direction. We assume that the vehicle drives in a straight line, and the equation for the hypothetical driving track is shown in the following formula:

$$
\begin{gathered}
\text { longitudinal range } \in[4 m, 21 m] \\
\text { lateral range }=-2 m
\end{gathered}
$$

The $2 \mathrm{D}$ road height profile, whose accuracy should correspond with that of the $3 \mathrm{D}$ point cloud, is discrete because the 3D point cloud data is discrete. Therefore, we should calculate the accuracy and then extract the road height profile according to the calculated precision.

Since the resolution of point cloud is affected by many factors, such as the number of lasers in LiDAR and vehicle speed, the average resolution of point cloud is used in the subsequent calculation.

The volume of the denoised 3D point cloud $V$ and the single point occupancy volume $v$ can be written as follows:

$$
\begin{aligned}
V & =\text { range }_{x} \times \text { range }_{y} \times \text { range }_{z} \\
\text { range }_{x} & =20.9994-4.0003=16.9991 \\
\text { range }_{y} & =2.9998+4.9935=7.99330 \\
\text { range }_{z} & =0.1977+0.1477=0.34540 \\
v & =\frac{V}{\text { the number of } 3 D \text { point cloud }}
\end{aligned}
$$

We regard $v$ as the cube box. Thus, the average resolution $d$ of the 3D point cloud can be considered as the length of the cube. The calculation shows that the value of $d$ is approximately $5 \mathrm{~cm}$.

IDW interpolation is a frequently used technique for LiDAR data [23]. The core idea of this technique is that nearby points are more alike than distant ones are. The weights assigned to the points closer to the prediction location are greater than those far away. It is calculated by using the following equation:

$$
\widehat{h}=\frac{\sum_{i=1}^{n}\left(1 / d_{i}\right) h_{i}}{\sum_{i=1}^{n}\left(1 / d_{i}\right)}
$$

where $h_{i}$ is the known height value and $d_{i}$ is the distance to the known points.

According to the precision of the denoised point cloud, a point to be interpolated is selected every $5 \mathrm{~cm}$ from the vehicle trajectory. Six nearest neighbours to that point are selected to interpolate using IDW with the weight of $1 / d_{i}$. Thereafter, the road height profile is obtained (Figure 7 ). The key road points of the vehicle's trajectory, which not only effectively reduce the number of point clouds but also improve the calculation speed, are thus obtained. These points are vital for the next step.

\section{Interpolation of Road Height Profile Based on Fractal Theory}

The preview sensor must be able to image sudden obstacles such as the high curb stone of standard EU 1340 and DIN 483. For this, the preview sensor has to scan the road with a grid width of $2 \mathrm{~cm}$. So an interpolation method close to the nature geometry should be selected to improve the accuracy of the road height profile, thereby enabling precise control of the active suspension.

Fractal interpolation, which is based on fractal theory, irregularity, roughness, and self-similarity of the dataset itself, uses the iterated function system (IFS) to focus on the retention of the global characteristics of a dataset [24, 25]. Recently, several studies have been including fractal compression and fractal interpolation methods in many digitised terrain reconstruction applications [26, 27]. Fractal interpolation can describe natural phenomena more realistically than can classic interpolation methods $[25,28]$. This theory utilises its similarity principle to observe detailed structures hidden in a chaotic appearance as a thinking tool for the understanding of global appearance from local characteristics [28]. Figure 8 shows the difference between traditional and fractal geometries on data simulation. Fractal geometry can express the details of a simulated object, whereas traditional geometry cannot [29]. The calculation of fractal interpolation is described in Section 10 "Methodology."

6.1. Quantitative Analysis of Road Height Profile's Fractal Characteristics. The quantitative analysis of fractal characteristics uses the "box covering method" to obtain the dual 


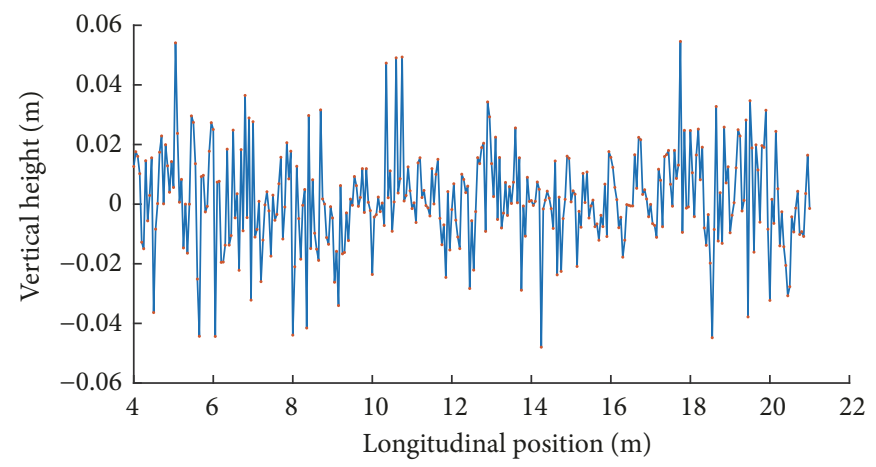

Figure 7: Road height profile using IDW algorithm.
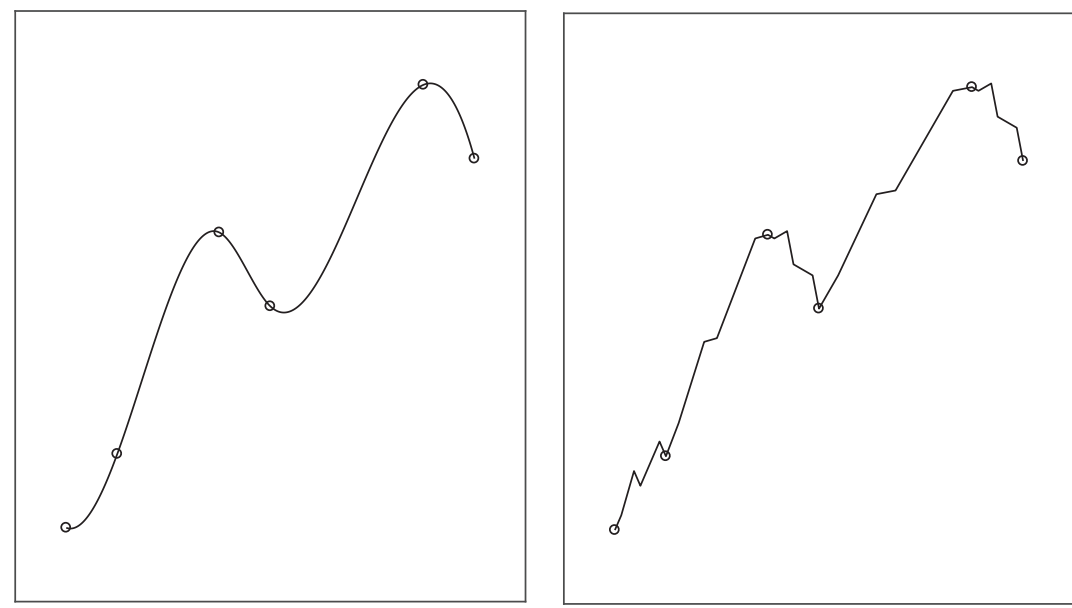

- Data Point
— Simulate Curve

- Data Point

- Simulate Curve

(a)

(b)

Figure 8: Data interpolation. (a) Spline interpolation. (b) Fractal interpolation.

TABLE 4: Coverage statistics.

\begin{tabular}{lccccc}
\hline$r_{i}(\mathrm{~m})$ & 0.005 & 0.01 & 0.02 & 0.04 & 0.08 \\
\hline$N\left(r_{i}\right)$ & 71400 & 18700 & 5100 & 1275 & 426 \\
\hline $\ln \left(1 / r_{i}\right)$ & 5.2983 & 4.6052 & 3.9120 & 3.2189 & 2.5257 \\
\hline $\ln \left(1 / N\left(r_{i}\right)\right)$ & 11.1761 & 9.8363 & 8.5370 & 7.1507 & 6.0544 \\
\hline
\end{tabular}

logarithmic coordinate diagram and to analyse its properties further. The calculation steps are as follows:

(1) The square with a length $r$ that covers the fractal curve is used (Figure 7).

(2) For different $r$, counting the number of squares $N(r)$ is to cover the fractal curve.

(3) The dual logarithmic coordinate diagram of $\ln N(r)-$ $\ln (1 / r)$ is plotted.

(4) The correlation coefficient of discrete points in the dual logarithmic coordinate diagram is calculated.

Table 4 lists the values of $r_{i}, N\left(r_{i}\right), \ln \left(1 / r_{i}\right)$, and $\ln N\left(r_{i}\right)$.
The data of $\ln \left(1 / r_{i}\right)$ and $\ln \left(1 / N\left(r_{i}\right)\right)$ are fitted by using the least squares method. Thus, a dual logarithmic regression line graph is obtained (Figure 9). The regression linear equation is as follows:

$$
\ln N\left(r_{i}\right)=1.8652 \cdot \ln \left(\frac{1}{r_{i}}\right)+1.2541
$$

The correlation coefficient $R$ of discrete points $\ln \left(1 / N\left(r_{i}\right)\right)$ and $\ln \left(1 / r_{i}\right)$ is as follows:

$$
R=99.94 \%
$$

where $R=99.94 \%$ indicates that the road height profile has strong self-similarity. It also reflects the fractal characteristics of the curve quantitatively. Therefore, the theory and method of fractal interpolation can be used to study Figure 7 .

6.2. Fractal Interpolation of Road Height Profile. The data obtained from Section 5.2 are interpolated by fractal interpolation. Four data points are inserted between each interval, 


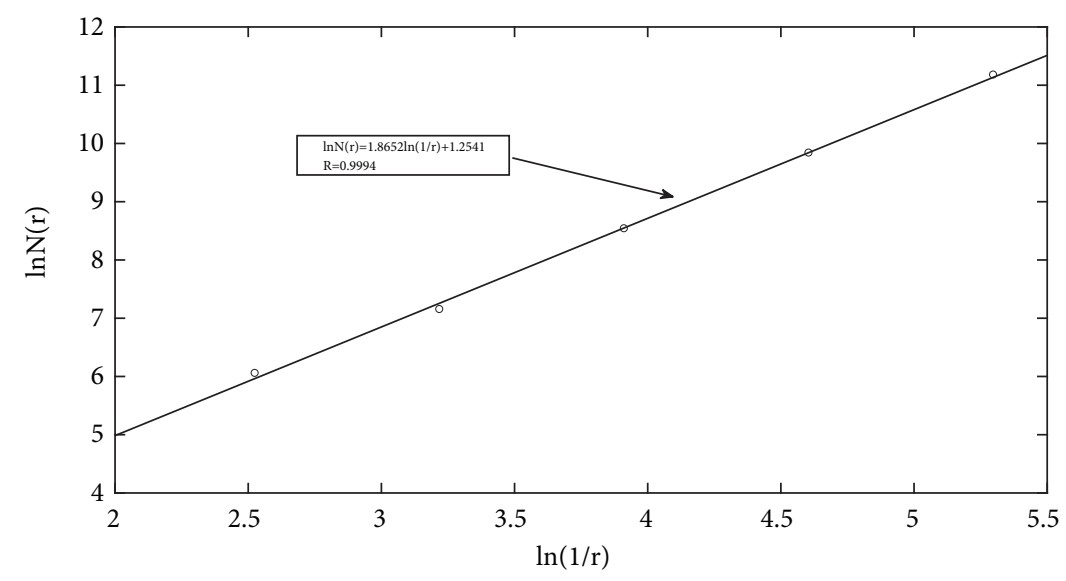

FIGURE 9: Double logarithmic map of road height profile based on box covering method.

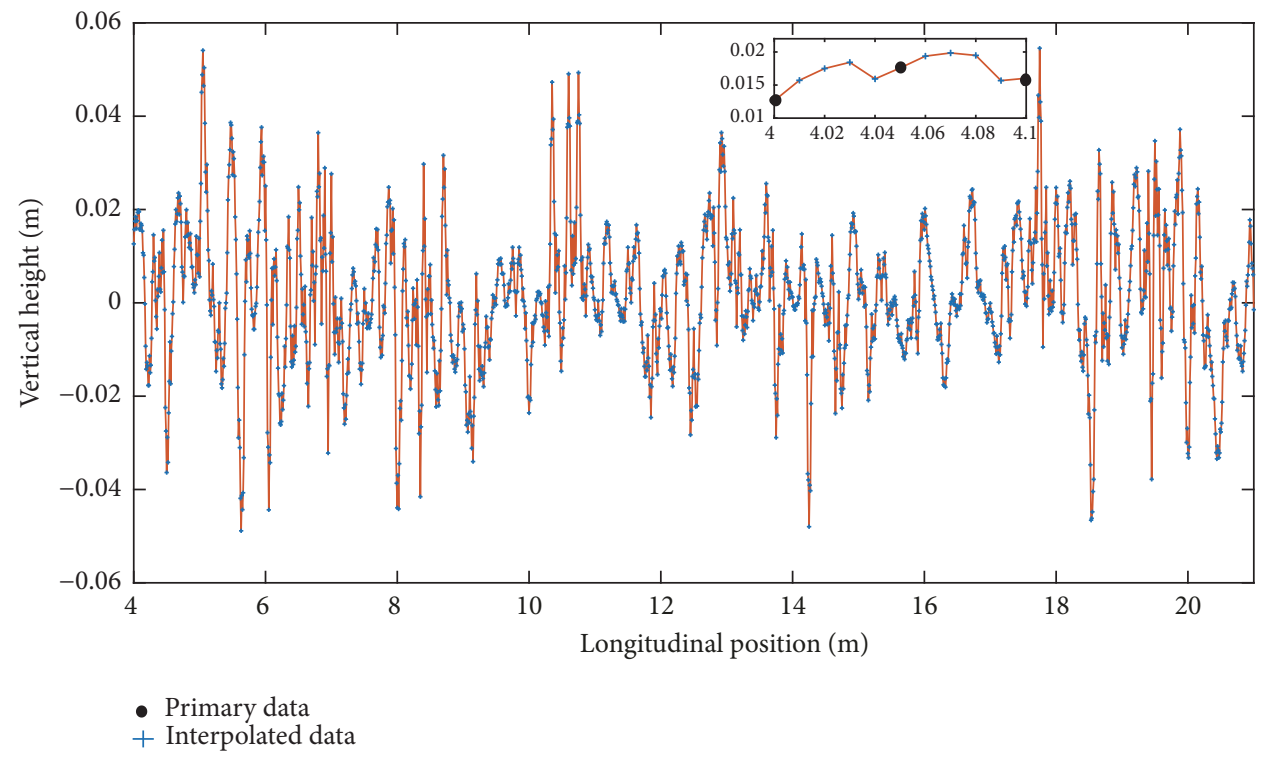

FIGURE 10: Road height information after fractal interpolation.

which is equivalent to obtaining a road height profile every 1 $\mathrm{cm}$. Figure 10 shows the road height information after fractal interpolation.

\section{Roughness Grade Division of Road Height Information}

Vehicle suspension is actively controlled using the LQG controller to enable the vehicle to obtain improved ride comfort and handling stability on the basis of road height information. Therefore, suitable LQG controller's weight coefficients of performance indicators that vary in different road roughness grades should be selected. The International Organisation for Standardisation (ISO 8608, 2016) proposed a methodology for classifying road profiles on the basis of power spectral density [30], which divides road roughness into eight grades $(A-H)$.
The road height information must be initially divided. The road surface roughness index unifies the traditional statistical parameter and the fractal dimension and is thus suitable for the grade of the road surface [31]. In [31], the expression of road roughness index is as follows:

$$
R^{*}=R_{q}^{1 /(D-1)}
$$

where $D$ and $R_{q}$ are the fractal dimension and root mean square (RMS) error of the road surface, respectively. The road height information in this study is as follows:

$$
\begin{aligned}
R_{q} & =15.7037 \times 10^{-3} \mathrm{~m} \\
D & =1.8652 \\
R^{*} & =24.1180 \times 10^{-3} \mathrm{~m}
\end{aligned}
$$




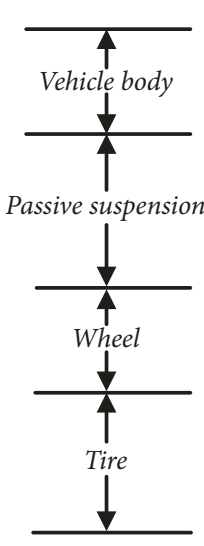

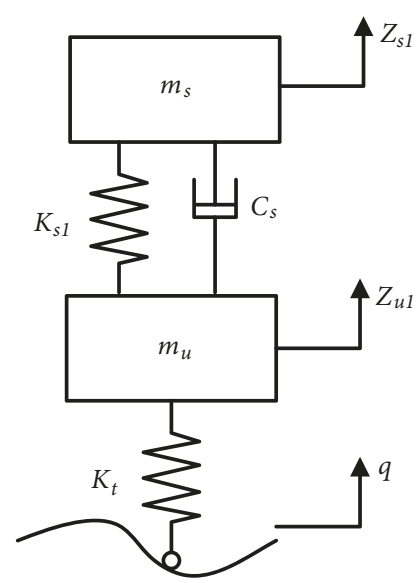

(a)

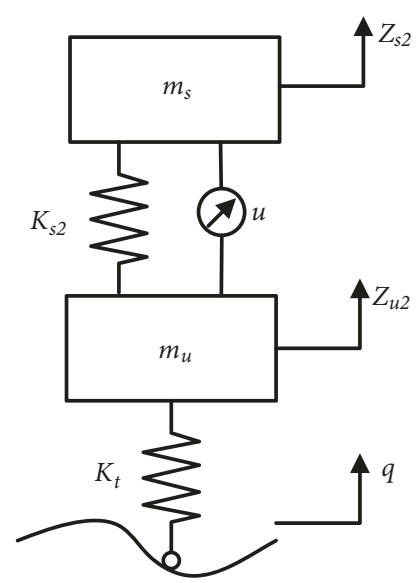

(b)

FIgURE 11: Quarter vehicle model. (a) Passive suspension. (b) Active suspension.

TABLE 5: Quarter vehicle model parameters.

\begin{tabular}{|c|c|c|c|}
\hline Symbol & Quantity & Value & Unit \\
\hline$m_{s}$ & Sprung mass & 320 & $\mathrm{~kg}$ \\
\hline$m_{u}$ & Unsprung mass & 40 & $\mathrm{~kg}$ \\
\hline$K_{s 1}$ & Passive suspension stiffness & 22000 & $N / m$ \\
\hline$K_{s 2}$ & Active suspension stiffness & 20000 & $N / m$ \\
\hline$K_{t}$ & Tire stiffness & 200000 & $N / m$ \\
\hline$C_{s}$ & Passive suspension damping & 1000 & $N \cdot s / m$ \\
\hline$Z_{s 1}$ & Sprung mass's displacements of passive suspension & - & $m$ \\
\hline$Z_{s 2}$ & Sprung mass's displacements of active suspension & - & $m$ \\
\hline$Z_{u 1}$ & Unsprung mass's displacements of passive suspension & - & $m$ \\
\hline$Z_{u 2}$ & Unsprung mass's displacements of active suspension & - & $m$ \\
\hline$q$ & Displacements of road excitation & - & $m$ \\
\hline$u$ & Active force & - & $N$ \\
\hline
\end{tabular}

according to the data provided in [31], which belongs to $B$ grade. Next, the LQG controller of the square active suspension is designed to solve the controller's weight coefficients under different road roughness grades.

\section{Optimal Design of LQG Controller}

Before designing the LQG controller of the active suspension, a dynamic model of passive and active suspensions of the vehicle, which is the basis of follow-up calculation, must be established.

8.1. Suspension System Dynamics. Figure 11 establishes a quarter vehicle model with passive and active suspensions, which have two degrees of freedom and heave dynamics. Unlike passive suspension, active suspension has only one input of road excitation and more active controlling force; moreover, a force actuator is installed in place of the damper variable. In this implementation, the tire is represented by a linear spring. In Table 5, the parameters of the quarter vehicle model are shown. Since the active suspension is a parallel structure of actuator and spring, the passive suspension replaces actuator with damper, so the suspension stiffness of both is set to different values.

According to the quarter vehicle model based on Newton's second law, the equations of motion of the system are as follows [32]:

(1) Passive suspension:

$$
\begin{aligned}
m_{u} \ddot{Z}_{u 1}= & K_{t}\left(q-Z_{u 1}\right)-K_{s 1}\left(Z_{u 1}-Z_{s 1}\right) \\
& -C_{S}\left(\dot{Z}_{u 1}-\dot{Z}_{s 1}\right) \\
m_{s} \ddot{Z}_{s 1}= & K_{s 1}\left(Z_{u 1}-Z_{s 1}\right)+C_{S}\left(\dot{Z}_{u 1}-\dot{Z}_{s 1}\right)
\end{aligned}
$$

(2) Active suspension:

$$
\begin{aligned}
& m_{u} \ddot{Z}_{u 2}=K_{t}\left(q-Z_{u 2}\right)-K_{s 1}\left(Z_{u 2}-Z_{s 2}\right)-u \\
& m_{s} \ddot{Z}_{s 2}=K_{s 1}\left(Z_{u 2}-Z_{s 2}\right)+u
\end{aligned}
$$


The state equation is established, and the system state variable $X$ is defined as follows:

$$
\begin{aligned}
& X_{1}=\left[\begin{array}{llll}
\dot{Z}_{s 1} & \dot{Z}_{u 1} & Z_{s 1} & Z_{u 1}
\end{array}\right]^{T} \\
& X_{2}=\left[\begin{array}{llll}
\dot{Z}_{s 2} & \dot{Z}_{u 2} & Z_{s 2} & Z_{u 2}
\end{array}\right]^{T}
\end{aligned}
$$

The suspension system can be expressed in the following state equation:

$$
\begin{aligned}
\dot{X}_{1} & =A_{1} \cdot X_{1}+B_{1} \cdot q \\
\dot{X}_{2} & =A_{2} \cdot X_{2}+B_{2} \cdot U \\
U & =\left[\begin{array}{l}
u \\
q
\end{array}\right]
\end{aligned}
$$

where

$$
\begin{aligned}
& A_{1}=\left[\begin{array}{cccc}
-\frac{C_{s}}{m_{s}} & \frac{C_{s}}{m_{s}} & -\frac{K_{s 1}}{m_{s}} & \frac{K_{s 1}}{m_{s}} \\
\frac{C_{s}}{m_{u}} & -\frac{C_{s}}{m_{u}} & \frac{K_{s 1}}{m_{u}} & -\frac{K_{s 1}+K_{t}}{m_{u}} \\
1 & 0 & 0 & 0 \\
0 & 1 & 0 & 0
\end{array}\right] \\
& B_{1}=\left[\begin{array}{c}
0 \\
\frac{K_{t}}{m_{u}} \\
0 \\
0
\end{array}\right] \\
& A_{2}=\left[\begin{array}{cccc}
0 & 0 & -\frac{K_{s 2}}{m_{s}} & \frac{K_{s 2}}{m_{s}} \\
0 & 0 & \frac{K_{s 2}}{m_{u}} & -\frac{K_{s 2}+K_{t}}{m_{u}} \\
1 & 0 & 0 & 0 \\
0 & 1 & 0 & 0
\end{array}\right] \\
& B_{2}=\left[\begin{array}{cc}
\frac{1}{m_{s}} & 0 \\
-\frac{1}{m_{u}} & \frac{K_{t}}{m_{u}} \\
0 & 0 \\
0 & 0
\end{array}\right]
\end{aligned}
$$

The system output $Y$ is defined as follows:

$$
\begin{aligned}
& Y_{1}=\left[\begin{array}{lll}
\ddot{Z}_{s 1} & Z_{u 1}-Z_{s 1} & q-Z_{u 1}
\end{array}\right]^{T} \\
& Y_{2}=\left[\begin{array}{lll}
\ddot{Z}_{s 2} & Z_{u 2}-Z_{s 2} & q-Z_{u 2}
\end{array}\right]^{T}
\end{aligned}
$$

The output equation of the system is as follows:

$$
\begin{aligned}
& Y_{1}=C_{1} \cdot X+D_{1} \cdot q \\
& Y_{2}=C_{2} \cdot X+D_{2} \cdot U
\end{aligned}
$$

where

$$
C_{1}=\left[\begin{array}{cccc}
-\frac{C_{s}}{m_{s}} & \frac{C_{s}}{m_{s}} & -\frac{K_{s 1}}{m_{s}} & \frac{K_{s 1}}{m_{s}} \\
0 & 0 & -1 & 1 \\
0 & 0 & 0 & -1
\end{array}\right]
$$$$
D_{1}=\left[\begin{array}{l}
0 \\
0 \\
1
\end{array}\right]
$$$$
C_{2}=\left[\begin{array}{cccc}
0 & 0 & -\frac{K_{s 2}}{m_{s}} & \frac{K_{s 1}}{m_{s}} \\
0 & 0 & -1 & 1 \\
0 & 0 & 0 & -1
\end{array}\right]
$$

$$
D_{2}=\left[\begin{array}{cc}
\frac{1}{m_{s}} & 0 \\
0 & 0 \\
0 & 1
\end{array}\right]
$$

8.2. LQG Controller. The main performance evaluation indicators in designing the controller for the quarter active suspension are as follows: (1) vehicle body acceleration, which evaluates ride comfort and handing stability; (2) suspension dynamic deflection, which affects body posture; and (3) tire dynamic deflection on behalf of the tire road holding [33].

Here, the comprehensive performance evaluation indicator denoted by $J$ is defined as follows $[34,35]$ :

$$
\begin{aligned}
J= & \lim _{T \rightarrow \infty} \frac{1}{T}\left\{q_{1} \cdot\left[Z_{u 2}(t)-q\right]^{2}+q_{2}\right. \\
& \left.\cdot\left[Z_{s 2}(t)-Z_{u 2}(t)\right]^{2}+q_{3} \cdot \ddot{Z}_{s 2}{ }^{2}(t)\right\} d t
\end{aligned}
$$

where $q_{1}, q_{2}, q_{3}$ are the weight coefficients of tire dynamic deflection, suspension dynamic deflection, and vehicle body acceleration, respectively. The weight coefficient $q_{3}$ of the vehicle body acceleration, which is used as a base value for the other coefficients to simplify the calculation, is set to 1 because it is important for vehicle body acceleration.

Then, formula (25) can be rewritten as an integral type of quadratic function by the following:

$$
J=\lim _{T \rightarrow \infty} \frac{1}{T}\left\{X_{2}{ }^{T} Q X_{2}+U^{T} R U+2 X_{2}^{T} N U\right\} d t
$$

where

$$
Q=\left[\begin{array}{cccc}
0 & 0 & 0 & 0 \\
0 & 0 & 0 & 0 \\
0 & 0 & q_{2}+\frac{q_{3} K_{s 2}^{2}}{m_{s}^{2}} & -q_{2}-q_{3} \frac{K_{s 2}^{2}}{m_{s}^{2}} \\
0 & 0 & -q_{2}-q_{3} \frac{K_{s 2}^{2}}{m_{s}^{2}} & q_{1}+q_{2}+q_{3} \frac{K_{s 2}^{2}}{m_{s}^{2}}
\end{array}\right]
$$




$$
\begin{aligned}
& N=\left[\begin{array}{cc}
0 & 0 \\
0 & 0 \\
-\frac{K_{s 2}}{m_{s}^{2}} & 0 \\
\frac{K_{s 2}}{m_{s}^{2}} & -q_{1}
\end{array}\right] \\
& R=\left[\begin{array}{cc}
\frac{q_{3}}{m_{s}^{2}} & 0 \\
0 & q_{1}
\end{array}\right]
\end{aligned}
$$

When the vehicle parameter values and weight coefficients $q_{1}, q_{2}, q_{3}$ are determined, the optimal control feedback gain matrix $K$ can be obtained from the following Riccati equation:

$$
\begin{aligned}
& P A_{2}+A_{2}^{T} P-\left(P B_{2}+N\right) R^{-1}\left(B_{2}^{T} P+N^{T}\right)+Q=0 \\
& K=R^{-1}\left(B_{2}^{T} P+N^{T}\right)
\end{aligned}
$$

Then, according to the state variables $X_{2}$ at any time, the optimal control force of the actuator denoted by $u$ can be described as follows:

$$
u=-K \cdot X_{2}
$$

The solving method of the weight coefficients in different road grades is discussed in the succeeding section.

\section{Solution of Weight Coefficients}

We need to solve eight sets of weight coefficients to enable the active suspension to adapt to different road grades. According to [36], random road excitation can be written as follows:

$$
\dot{q}(t)=-2 \pi n_{00} v q(t)+2 \pi n_{0} \sqrt{S_{q}\left(n_{0}\right) v} W(t)
$$

where $n_{00}=0.011 \mathrm{~m}^{-1}$ is the spatial lower cut-off frequency. $W(t)$ stands for Gaussian white noise with zero mean. $v$ represents the speed of the vehicle.

According to formula (31), under the speed $40 \mathrm{~km} / \mathrm{h}$, we obtain eight grades of road surface roughness, as shown in Figure 12.

AHP is a multicriteria decision-aiding method that is based on a solid axiomatic foundation. It is a systematic procedure for dealing with complex decision-making problems, in which many competing alternatives exist [37-39]. Thus, we adopt this method to solve the weight coefficients of the LQG controller. The process of calculating LQG controller coefficients using AHP method is described in Section 10 "Methodology."

Table 6 lists the passive suspension performance statistics under different road grades.

On the high-grade road surface, the ride comfort is mainly optimized, and on the poor grade road surface,

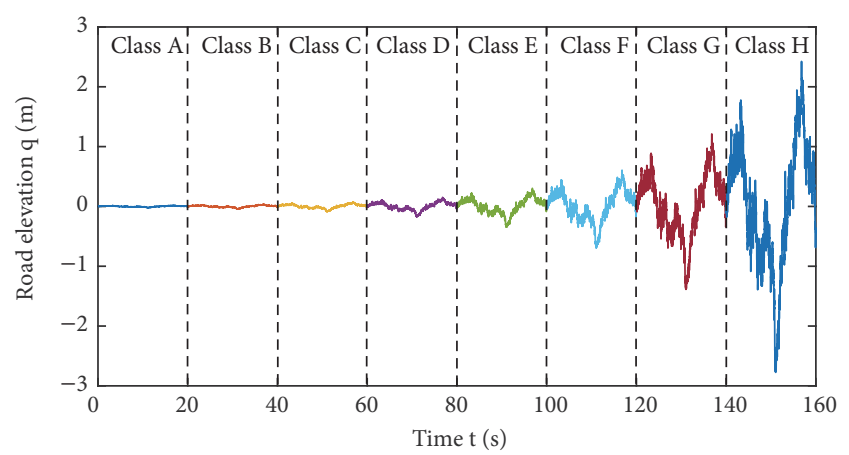

FIGURE 12: Road surface roughness of different grades.

TABle 6: Performance statistics of passive suspension under different road grades.

\begin{tabular}{lccc}
\hline Road grades & $\sigma_{\text {BA1 }}\left(\mathrm{m} / \mathrm{s}^{2}\right)$ & $\sigma_{\text {DTD1 }}(\mathrm{m})$ & $\sigma_{\text {SWS1 }}(\mathrm{m})$ \\
\hline $\mathrm{A}$ & 0.3418 & 0.0011 & 0.0034 \\
\hline $\mathrm{B}$ & 0.6836 & 0.0023 & 0.0069 \\
\hline $\mathrm{C}$ & 1.3671 & 0.0046 & 0.0137 \\
\hline $\mathrm{D}$ & 2.7342 & 0.0091 & 0.0275 \\
\hline $\mathrm{E}$ & 5.4684 & 0.0182 & 0.0550 \\
\hline $\mathrm{F}$ & 10.9368 & 0.0365 & 0.1099 \\
\hline $\mathrm{G}$ & 21.8737 & 0.0729 & 0.2198 \\
\hline $\mathrm{H}$ & 43.7473 & 0.1458 & 0.4396 \\
\hline
\end{tabular}

the handling stability is mainly optimized. The subjective judgment matrices of different road grades are as follows:

$H_{A}=\left[\begin{array}{ccc}1 & 2 & 9 \\ \frac{1}{2} & 1 & 3 \\ \frac{1}{9} & \frac{1}{3} & 1\end{array}\right]$

$H_{B}=\left[\begin{array}{ccc}1 & 3 & 9 \\ \frac{1}{3} & 1 & 3 \\ \frac{1}{9} & \frac{1}{3} & 1\end{array}\right]$

$H_{C}=\left[\begin{array}{ccc}1 & \frac{3}{4} & 8 \\ \frac{4}{3} & 1 & 4 \\ \frac{1}{8} & \frac{1}{4} & 1\end{array}\right]$

$H_{D}=\left[\begin{array}{ccc}1 & \frac{3}{4} & 6 \\ \frac{4}{3} & 1 & 4 \\ \frac{1}{6} & \frac{1}{4} & 1\end{array}\right]$ 


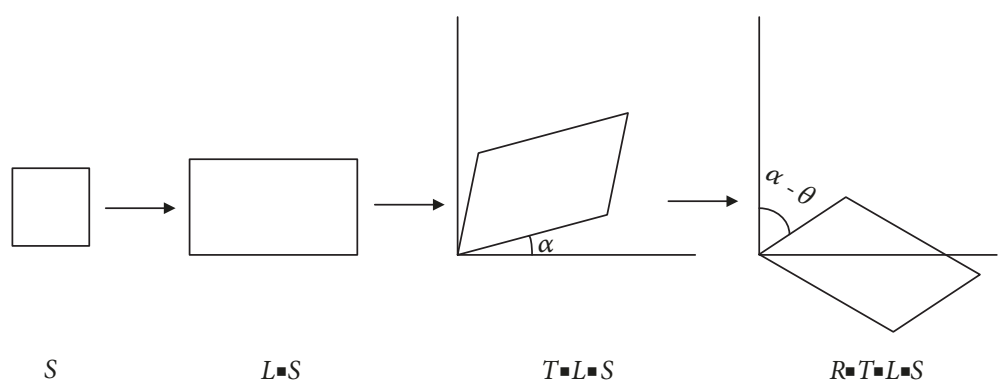

Figure 13: Affine transform decomposition diagram.

TABLE 7: LQG controller weighting factor in different road grades.

\begin{tabular}{lcc}
\hline Road grades & $q_{1}$ & $q_{2}$ \\
\hline $\mathrm{A}(\mathrm{qA})$ & 39323.4009 & 1259.5536 \\
\hline $\mathrm{B}(\mathrm{qB})$ & 30009.3714 & 1100.3214 \\
\hline $\mathrm{C}(\mathrm{qC})$ & 86562.0060 & 1716.5706 \\
\hline $\mathrm{D}(\mathrm{qD})$ & 95273.8155 & 2079.4772 \\
\hline $\mathrm{E}(\mathrm{qE})$ & 62422.0080 & 4760.8143 \\
\hline $\mathrm{F}(\mathrm{qF})$ & 71455.3616 & 6238.4316 \\
\hline $\mathrm{G}(\mathrm{qG})$ & 52648.7606 & 16933.7086 \\
\hline $\mathrm{H}(\mathrm{qH})$ & 49544.3806 & 17994.7505 \\
\hline
\end{tabular}

$$
\begin{aligned}
& H_{E}=\left[\begin{array}{lll}
1 & 1 & 3 \\
1 & 1 & 1 \\
\frac{1}{3} & 1 & 1
\end{array}\right] \\
& H_{F}=\left[\begin{array}{lll}
1 & 1 & 2 \\
1 & 1 & 1 \\
1 & 1 & 1
\end{array}\right] \\
& H_{G}=\left[\begin{array}{lll}
1 & 1 & 1 \\
1 & 1 & \frac{1}{5} \\
1 & 5 & 1
\end{array}\right] \\
& H_{H}=\left[\begin{array}{lll}
1 & 1 & 1 \\
1 & 1 & \frac{1}{6} \\
1 & 6 & 1
\end{array}\right]
\end{aligned}
$$

Table 7 shows the calculated LQG controller's weight coefficients in different road roughness grades.

Because the road height profile calculated in Section 7 belongs to $B$ grade, the weight coefficients in the LQG controller are selected as follows:

$$
\begin{aligned}
& q_{1}=30009.3714 \\
& q_{2}=1100.3214
\end{aligned}
$$

\section{Methodology}

\subsection{Fractal Interpolation}

10.1.1. Affine Transformation. A two-dimensional affine transformation $W$ is formed as follows:

$$
W\left[\begin{array}{l}
x^{\prime} \\
y^{\prime}
\end{array}\right]=\left[\begin{array}{ll}
a & b \\
c & d
\end{array}\right]\left[\begin{array}{l}
x \\
y
\end{array}\right]+\left[\begin{array}{l}
e \\
f
\end{array}\right]
$$

where $a, b, c, d, e, f$ are real numbers, $p(x, y)$ is a point on the plane, and $W$ is a linear affine transformation.

The transformation matrix $A$ can be decomposed into rotation $R$, expansion $L$, and distortion $T$.

$$
\begin{aligned}
A & =\left[\begin{array}{ll}
a & b \\
c & d
\end{array}\right]=R \cdot T \cdot L \\
R & =\left[\begin{array}{cc}
\cos \theta & -\sin \theta \\
\sin \theta & \cos \theta
\end{array}\right] \\
T & =\left[\begin{array}{cc}
1 & \tan \alpha \\
\tan \alpha & 1
\end{array}\right] \\
L & =\left[\begin{array}{ll}
l_{1} & 0 \\
0 & l_{2}
\end{array}\right]
\end{aligned}
$$

Figure 13 shows a decomposition diagram of an affine transformation.

10.1.2. IFS and Fractal Interpolation. A set of data points $\left\{P_{i}=\right.$ $\left.\left(x_{i}, y_{i}\right) \in R^{2} \mid i=0,1,2, \cdots, N\right\}$ satisfies $x_{0}<x_{1}<x_{2}<$ $\cdots<x_{N}$. The attractor of IFS is a continuous function of interpolating data. An interpolation curve across a series of data points $P_{i}$ and map $P_{o}$ and $P_{N}$ to $P_{n-1}$ and $P_{n}$ is formed.

Considering IFS $\left\{R^{2} ; W_{n}, n=0,1,2, \cdots, N\right\}, W_{n}$ is an affine transformation with the following form:

$$
W_{n}\left[\begin{array}{l}
x \\
y
\end{array}\right]=\left[\begin{array}{ll}
a_{n} & 0 \\
c_{n} & d_{n}
\end{array}\right] \cdot\left[\begin{array}{l}
x \\
y
\end{array}\right]+\left[\begin{array}{l}
e_{n} \\
f_{n}
\end{array}\right]
$$


TABLE 8: Comparison values of importance between indicators.

\begin{tabular}{lccccc}
\hline$i / j$ & Equally & Moderately & Strongly & Very & Extremely \\
\hline$h_{i j}$ & 1 & 3 & 5 & 7 & 9 \\
\hline
\end{tabular}

The aforementioned equation satisfies the following:

$$
\begin{aligned}
W_{n}\left[\begin{array}{l}
x_{0} \\
y_{0}
\end{array}\right] & =\left[\begin{array}{l}
x_{n-1} \\
y_{n-1}
\end{array}\right] \\
W_{n}\left[\begin{array}{l}
x_{N} \\
y_{N}
\end{array}\right] & =\left[\begin{array}{l}
x_{n} \\
y_{n}
\end{array}\right]
\end{aligned}
$$

where $b_{n}=0$ guarantees that the interpolation function of each cell does not overlap. Assume that $d_{n}$ is constant; then we can solve the parameters as follows [38]:

$$
\begin{aligned}
& a_{n}=\frac{\left(x_{n}-x_{n-1}\right)}{\left(x_{N}-x_{0}\right)} \\
& e_{n}=\frac{\left(x_{N} x_{n-1}-x_{0} x_{n}\right)}{\left(x_{N}-x_{0}\right)} \\
& c_{n}=\frac{\left(y_{n}-y_{n-1}-d_{n}\left(y_{N}-y_{0}\right)\right)}{\left(x_{N}-x_{0}\right)} \\
& f_{n}=\frac{\left(x_{N} y_{n-1}-x_{0} y_{n}-d_{n}\left(x_{N} y_{0}-x_{0} y_{N}\right)\right)}{\left(x_{N}-x_{0}\right)}
\end{aligned}
$$

For a set of data points $\left\{P_{i}=\left(x_{i}, y_{i}\right) \in R^{2} \mid i=0,1,2, \cdots\right.$, $N\}, N$ numbers of affine transformations exist. The fractal interpolation function of the affine transformation of $n_{t h}$ is as follows:

$$
\begin{aligned}
& x_{n i}=a_{n} x_{i}+e_{n} \\
& y_{n i}=c_{n} x_{i}+d_{n} y_{i}+f_{n} \\
& \quad i=0,1, \cdots, N ; n=1,2, \cdots, N
\end{aligned}
$$

\subsection{Analytic Hierarchy Process}

10.2.1. Calculating Objective Weight Coefficient. According to the parameters of a passive suspension, its dynamic simulation model is operated under eight road grades to obtain the performance statistics $\sigma_{B A 1}, \sigma_{D T D 1}, \sigma_{S W S 1}$, which are the RMS of vehicle's vertical acceleration, tire dynamic deflection, and suspension dynamic deflection, respectively. The objective weight coefficient of vertical acceleration is supposed to be 1 . According to [38], the equation of calculating other objective weight coefficients is as follows:

$$
\sigma_{B A 1}^{2} \cdot 1=\beta_{1} \cdot \sigma_{D T D 1}^{2}=\beta_{2} \cdot \sigma_{S W S 1}^{2}
$$

10.2.2. Determining Subjective Weight Coefficients. (1) A subjective judgement matrix is created as follows.

$h_{i j}$ is the comparison value of the importance of the indicators $i$ and $j$, and a fundamental 1-to-9 scale can be used to rank the judgments (Table 8).
The subjective judgement matrix $H$ is built through pairwise comparison of each decision factor.

$$
H=\left[\begin{array}{ccccc}
1 & h_{12} & \cdots & \cdots & h_{1 n} \\
\frac{1}{h_{12}} & 1 & h_{23} & \cdots & h_{2 n} \\
\cdots & \frac{1}{h_{23}} & \cdots & \cdots & \cdots \\
\vdots & \vdots & \ddots & \ddots & \vdots \\
\frac{1}{h_{1 n}} & \frac{1}{h_{2 n}} & \cdots & \cdots & 1
\end{array}\right]
$$

(2) Matrix $H$ is calculated by multiplying the vector of every row as follows:

$$
\begin{aligned}
M & =\left[M_{1}, M_{2}, \cdots, M_{n}\right]^{T} \\
M_{i} & =\prod_{j=1}^{n} h_{i j} \\
& (i, j=1,2, \cdots, n)
\end{aligned}
$$

(3) $\sqrt[n]{M}$ is calculated as follows:

$$
\begin{aligned}
& \bar{W}=\left[\overline{W_{1}}, \overline{W_{2}}, \cdots, \overline{W_{n}}\right]^{T} \\
& \overline{W_{i}}=\sqrt[n]{M_{i}}
\end{aligned}
$$

$$
(i=1,2, \cdots, n)
$$

(4) Positive vector $W$ is calculated as follows:

$$
W=\frac{\bar{W}}{\sum_{i=1}^{n} \overline{W_{i}}}
$$

(5) The maximum eigenvalue of matrix $H$ is calculated and checked for consistency as follows.

If every element in matrix $H$ satisfies the equation $h_{i j}=$ $1 / h_{j i}$ and $h_{i j}=h_{i k} \bullet h_{k j}$, then the matrix is the consistency matrix. The subjective judgement matrix is often not perfectly consistent due to the randomness of people's judgment. These judgment errors can be detected by the consistency ratio $C R$.

$$
\begin{aligned}
\lambda_{\max } & =\sum_{i=1}^{n} \frac{(H W)_{i}}{n W_{i}} \quad(i=1,2, \cdots, n) \\
C R & =\frac{\lambda_{\max }-n}{R I(n-1)}
\end{aligned}
$$

where $R I$ is a random consistency index of matrix $H$.

When $n$ is $10, R I$ is equal to 1.4851 . The solution is correct when $C R$ is less than 1 . If $C R$ is more than 1 , then the evaluation matrix $H$ needs to be revised on the basis of [39].

(6) Subjective weight coefficients.

The subjective weight coefficient of the vehicle's vertical acceleration is 1 . The subjective weight coefficients of the tire dynamic deflection $\gamma_{1}$ and suspension dynamic deflection $\gamma_{2}$ are determined according to the following formula:

$$
W_{1}=\frac{W_{2}}{\gamma_{1}}=\frac{W_{3}}{\gamma_{2}}
$$




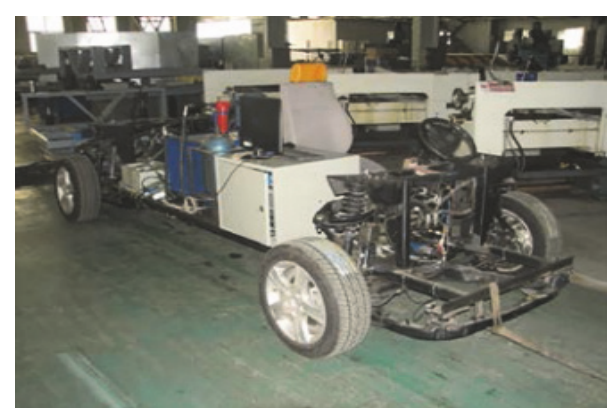

Figure 14: Prototype vehicle with active suspension.

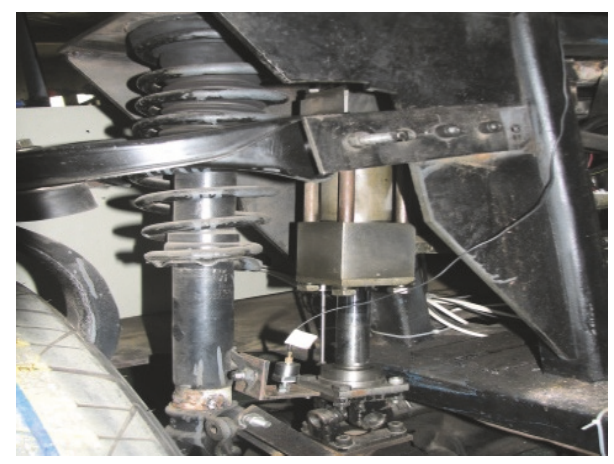

Figure 15: Single active suspension actuator.

10.2.3. Calculating LQG Controller's Weight Coefficients $q_{1}$ and $q_{2}$. The general weights of the evaluating indexes related to ride comfort can be obtained on the basis of the objective weights and subjective weight coefficients.

$$
\begin{aligned}
& q_{1}=\beta_{1} \cdot \gamma_{1} \\
& q_{2}=\beta_{2} \cdot \gamma_{2}
\end{aligned}
$$

\section{Experiment}

When the vehicle's speed is constant, the more point cloud data are collected and the higher data fusion rate is required. Therefore, in order to meet the needs of high-speed data processing of active suspension, firstly, only collect and store the topographic data in front of the vehicle; secondly, collect the data by using the suitable number of lines of LiDAR; thirdly, the algorithm adopted in this paper not only effectively collects enough point cloud data on the ground but also satisfies the accuracy of the solution after data fusion and obtains the elevation information of the road surface for effective control of suspension.

Road experiments of the prototype vehicle are performed to verify the accuracy and efficiency of the active suspension control algorithm that is based on LiDAR-previewed terrain information. The main body of the prototype vehicle is assembled and constructed using the FAW Besturn car chassis (Figure 14). It includes the mechanical, hydraulic servo, and the electric control parts. The single active suspension actuator unit is shown in Figure 15, and the left side is the servo control actuator cylinder. Figure 4 shows the test site.
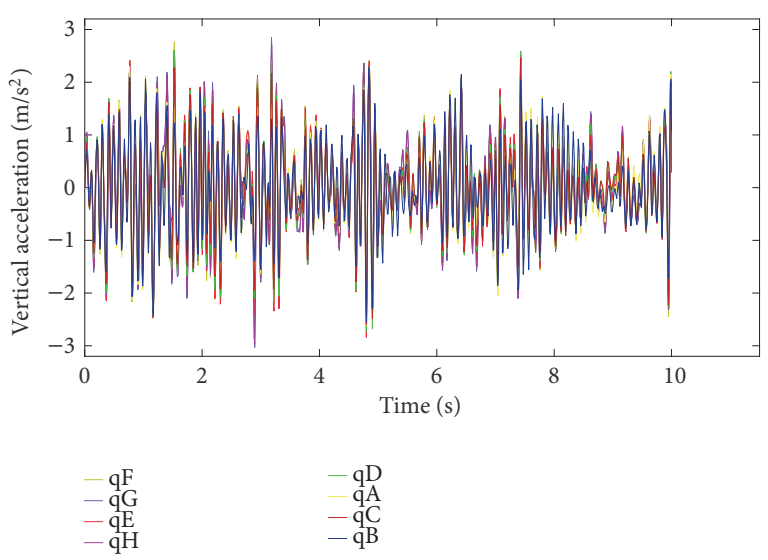

FIGURE 16: Vertical acceleration of active suspension.

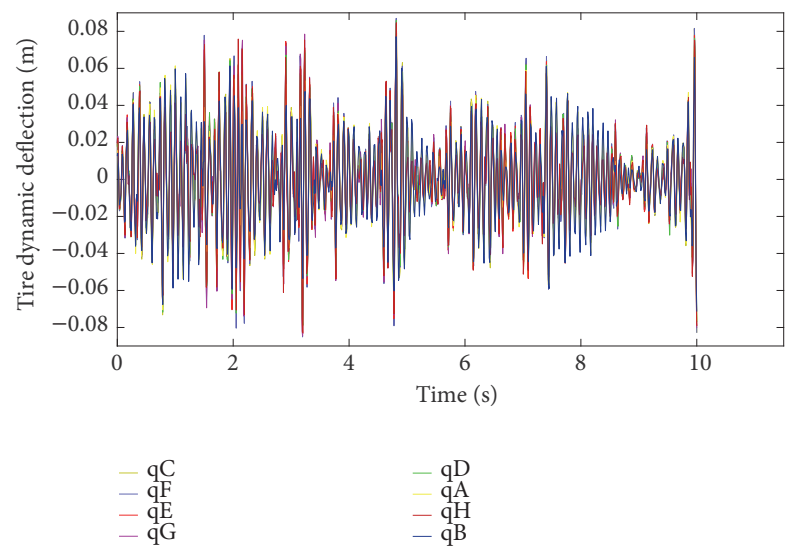

FIGURE 17: Tire dynamic deflection of active suspension.

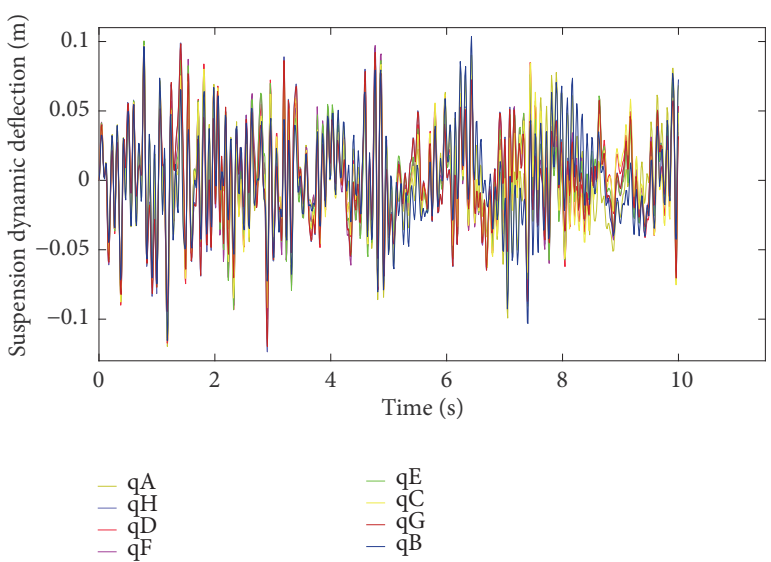

FIGURE 18: Active suspension dynamic deflection.

Figures 16-22 show the experiment data. In order to properly validate selection of control weightings based on estimated road profile and road class, the performance evaluation indicators of active control for different weight factors $\mathrm{q} 1$ and $\mathrm{q} 2$ that are under different road class are reported in Figures 16-18. Figures 19-21 depict the performance evaluation indicator comparison between active suspension 
TABLE 9: RMS values of active and passive suspension performance indicators.

\begin{tabular}{lccccccccc}
\hline & \multicolumn{1}{c}{ Active suspension } & \multicolumn{3}{c}{ Passive suspension } \\
& $q_{A}$ & $q_{B}$ & $q_{C}$ & $q_{D}$ & $q_{E}$ & $q_{F}$ & $q_{G}$ & $q_{H}$ \\
\hline Vertical acceleration $\left(m / s^{2}\right)$ & 0.8581 & 0.8277 & 0.8342 & 0.8909 & 0.9173 & 0.9231 & 0.9206 & 0.9028 \\
\hline Tire deflection $(m)$ & 0.0246 & 0.0242 & 0.0258 & 0.0247 & 0.0251 & 0.0256 & 0.0248 & 0.0245 & 0.0277 \\
\hline Suspension deflection $(m)$ & 0.0344 & 0.0335 & 0.0337 & 0.0342 & 0.0338 & 0.0339 & 0.0336 & 0.0343 & 0.0368 \\
\hline
\end{tabular}

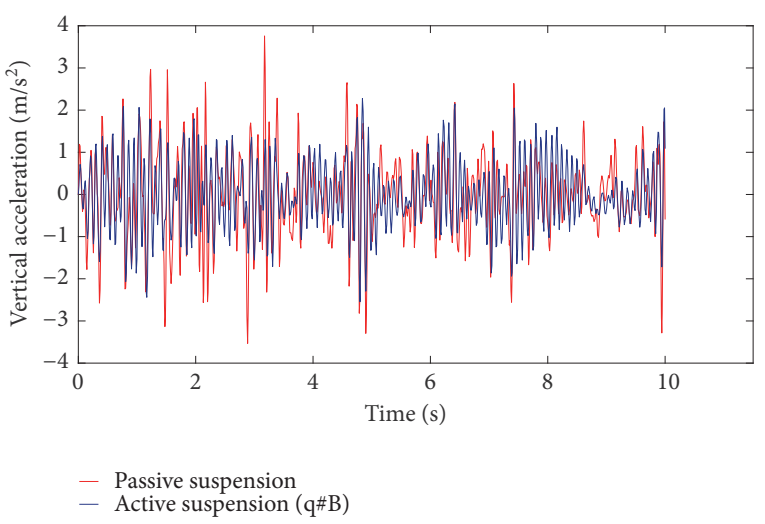

FIGURE 19: Vertical acceleration.

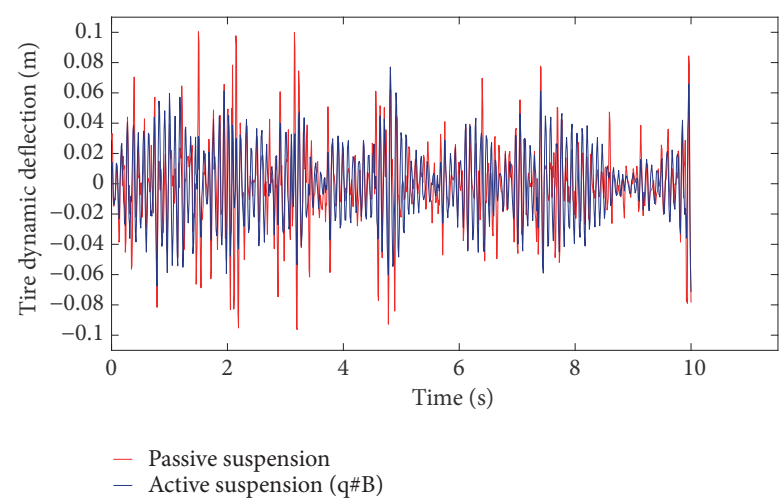

Figure 20: Tire dynamic deflection.

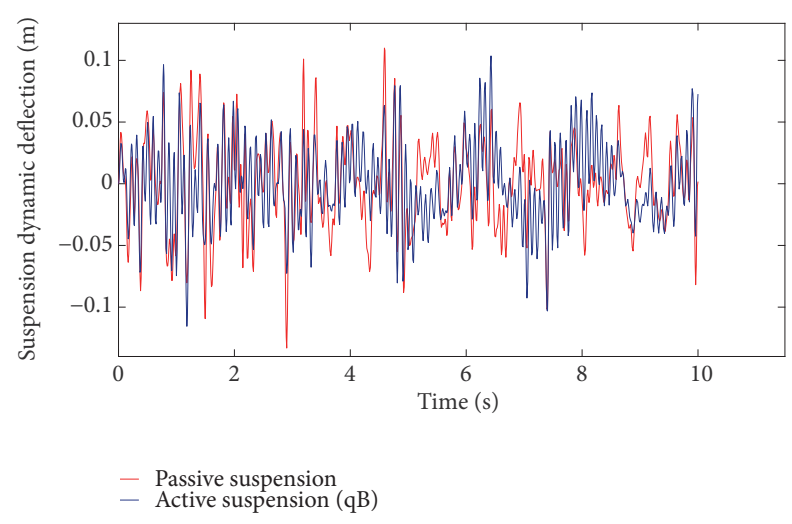

FIGURE 21: Suspension dynamic deflection.

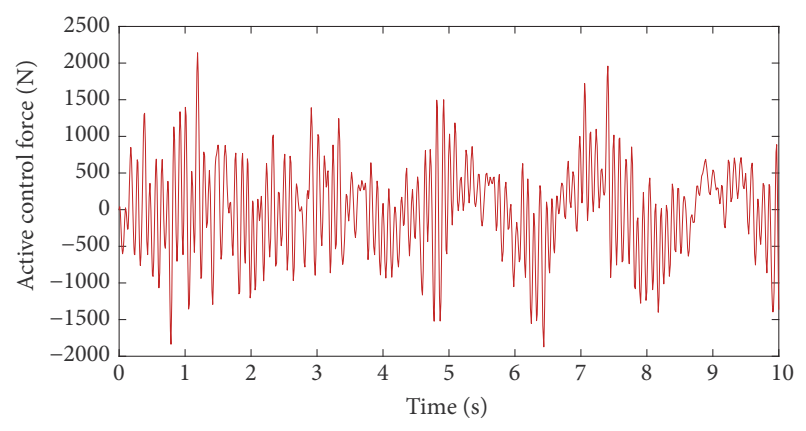

FIGURE 22: Active control force.

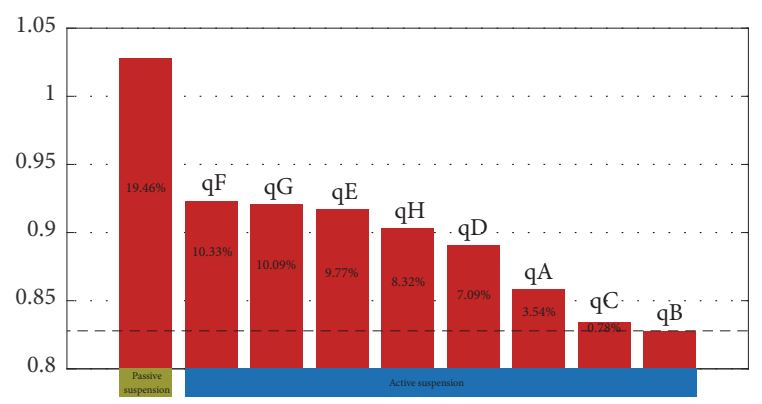

FIGURE 23: Bar-chart of suspension's vertical acceleration.

with LQG controller whose controller coefficient is selected as $\mathrm{qB}$ and passive suspension. Figure 22 shows changes in the control force of the active suspension actuators.

Table 9 shows the RMS of performance statistics of LQGactive suspension system based on different weight coefficients $\mathrm{q} 1$ and $\mathrm{q} 2$ and passive suspension system. Draw the data in Table 9 as a bar chart as shown in Figures 23-25. As can be seen from Figure 23, the RMS of vertical acceleration of LQG active suspension controller based on grade B pavement coefficients $\mathrm{qB}$ is about $19.46 \%$ lower than that of passive suspension and about $10.33 \%, 10.09 \%, 9.77 \%, 8.32 \%, 7.09 \%$, $3.54 \%$, and $0.78 \%$ lower than the LQG active suspension controller based on other pavement grade coefficients $\mathrm{qF}, \mathrm{qG}$, $\mathrm{qE}, \mathrm{qH}, \mathrm{qD}, \mathrm{qA}$, and $\mathrm{qC}$.

In Figure 24, the RMS of tire dynamic deflection of LQG active suspension controller based on grade $B$ pavement coefficients $\mathrm{qB}$ is about $15.9 \%$ lower than that of passive suspension and about $10.33 \%, 10.09 \%, 9.77 \%, 8.32 \%, 7.09 \%$, $3.54 \%$, and $0.78 \%$ lower than the LQG active suspension controller based on other pavement grade coefficients $\mathrm{qF}, \mathrm{qG}$, $\mathrm{qE}, \mathrm{qH}, \mathrm{qD}, \mathrm{qA}$, and $\mathrm{qC}$. 


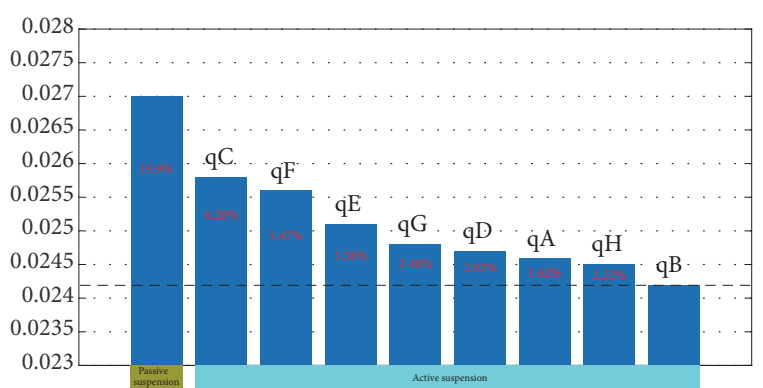

FIGURE 24: Bar-chart of suspension's tire dynamic deflection.

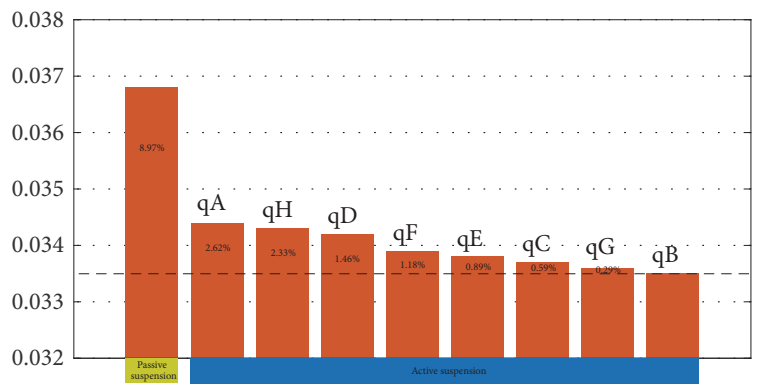

FIGURE 25: Bar-chart of suspension's dynamic deflection.

In Figure 25, the RMS of suspension dynamic deflection of LQG active suspension controller based on grade B pavement coefficients $\mathrm{qB}$ is about $8.97 \%$ lower than that of passive suspension and about $2.62 \%, 2.33 \%, 1.46 \%, 1.18 \%, 0.89 \%$, $0.59 \%$, and $0.29 \%$ lower than the LQG active suspension controller based on other pavement grade coefficients qA, $\mathrm{qH}, \mathrm{qD}, \mathrm{qF}, \mathrm{qE}, \mathrm{qC}$, and $\mathrm{qG}$.

The experimental data show that reasonable selection of LQG controller weightings based on estimated road profile and road class through fractal interpolation can improve vehicle ride comfort and handling stability effectively more than passive suspension does.

\section{Conclusions}

This study focuses on extracting a road height profile from the $3 \mathrm{D}$ point cloud data of the terrain in front of a vehicle as input to the suspension control model. Three innovations are mainly presented. Firstly, a LiDAR coupled with INS and GPS is used to measure the 3D point cloud of the terrain in front of the vehicle. Although many researchers have proposed the use of preview information for active suspension, they did not provide detailed descriptions and instead only presented concepts. Secondly, the road height profile is extracted using the IDW interpolation method. The main difference is that this method has been used for 3D digital terrain obtained by airborne LiDAR systems previously and is rarely used in a vehicle's LiDAR system to obtain preview information of a road. Thirdly, fractal interpolation is used to improve the accuracy of road height information. This interpolation method is close to natural geometry. Thus, the interpolated data in this work are more precise than traditionally interpolated data.

Road experiment verifies that reasonable selection of LQG controller weightings based on estimated road profile and road class through fractal interpolation can improve the ride comfort and handling stability of the vehicle more than passive suspension does.

\section{Data Availability}

All data generated or analysed during this study are included in this published article.

\section{Conflicts of Interest}

The authors declare that there are no conflicts of interest regarding the publication of this paper.

\section{Authors' Contributions}

Mingde Gong conceived the idea, designed the experiments, and wrote the paper; Haohao Wang helped with the algorithm and analysed the experimental data; Xin Wang collated and analysed the experimental data.

\section{Acknowledgments}

This work was supported by the National Key Research and Development Program of China (Grant no. 2016YFC080290 $0)$.

\section{References}

[1] C. Gohrle, A. Schindler, A. Wagner, and O. Sawodny, "Design and vehicle implementation of preview active suspension controllers," IEEE Transactions on Control Systems Technology, vol. 22, no. 3, pp. 1135-1142, 2014.

[2] M. H. Kim and S. B. Choi, "Estimation of road surface height for preview system using ultrasonic sensor," in Proceedings of the International Conference on Networking, Sensing, and Control, pp. 1-4, IEEE, 2016.

[3] F. Oniga and S. Nedevschi, "Processing dense stereo data using elevation maps: road surface, traffic isle, and obstacle detection," IEEE Transactions on Vehicular Technology, vol. 59, no. 3, pp. 1172-1182, 2010.

[4] A. Jaakkola, J. Hyyppä, H. Hyyppä, and A. Kukko, "Retrieval algorithms for road surface modelling using laser-based mobile mapping," Sensors, vol. 8, no. 9, pp. 5238-5249, 2008.

[5] J. Laurent, M. Talbot, and M. Doucet, "Road surface inspection using laser scanners adapted for the high precision measurements of large flat surfaces," in Proceedings of the International Conference on Recent Advances in 3-D Digital Imaging and Modeling, pp. 303-310, IEEE, 1997.

[6] J. J. Dawkins, D. M. Bevly, and R. L. Jackson, "Evaluation of fractal terrain model for vehicle dynamic simulations," Journal of Terramechanics, vol. 49, no. 6, pp. 299-307, 2012.

[7] M. Rahman and G. Rideout, "Using the lead vehicle as preview sensor in convoy vehicle active suspension control," Vehicle System Dynamics, vol. 50, no. 12, pp. 1923-1948, 2012. 
[8] B. Fu, L. Liu, and J. Bao, "GPS/INS/speed log integrated navigation system based on MAKF and priori velocity information," in Proceedings of the IEEE International Conference on Information and Automation, pp. 54-58, IEEE, 2014.

[9] I. Skog and P. Händel, "In-car positioning and navigation technologiesa survey," IEEE Transactions on Intelligent Transportation Systems, vol. 10, no. 1, pp. 4-21, 2009.

[10] A. Noureldin, T. B. Karamat, M. D. Eberts, and A. El-Shafie, "Performance enhancement of MEMS-based INS/GPS integration for low-cost navigation applications," IEEE Transactions on Vehicular Technology, vol. 58, no. 3, pp. 1077-1096, 2009.

[11] M. S. Grewal, L. R. Weill, and A. P. Andrews, "Global postioning systems, inertial navigation, and integration," Wiley Interdisciplinary Reviews Computational Statistics, vol. 3, no. 4, pp. 383384, 2007.

[12] H. Zhao, Z. Xiong, L. Shi, F. Yu, and J. Liu, "A robust filtering algorithm for integrated navigation system of aerospace vehicle in launch inertial coordinate," Aerospace Science and Technology, vol. 58, pp. 629-640, 2016.

[13] X. Ning, M. Gui, Y. Xu, X. Bai, and J. Fang, "INS/VNS/CNS integrated navigation method for planetary rovers," Aerospace Science and Technology, vol. 48, no. 1, pp. 102-114, 2015.

[14] M. Enkhtur, S. Y. Cho, and K. H. Kim, "Modified unscented kalman filter for a multirate INS/GPS integrated navigation system," ETRI Journal, vol. 35, no. 5, pp. 943-946, 2013.

[15] J. Landa, D. Procházka, and J. Št’astný, "Point cloud processing for smart systems," Acta Universitatis Agriculturae et Silviculturae Mendelianae Brunensis, vol. 61, no. 7, pp. 2415-2421, 2013.

[16] X. Han, J. S. Jin, M. Wang, W. Jiang, L. Gao, and L. Xiao, "A review of algorithms for filtering the 3D point cloud," Signal Processing: Image Communication, vol. 57, pp. 103-112, 2017.

[17] H. Xie, K. T. McDonnell, and H. Qin, "Surface reconstruction of noisy and defective data sets," in Proceedings of the IEEE Visualization 2004, pp. 259-266, IEEE, USA, 2004.

[18] J. Park, H. Kim, Y. W. Tai, M. S. Brown, and I. Kweon, "High quality depth map upsampling for 3D-TOF cameras," in Proceedings of the 2011 IEEE International Conference on Computer Vision, ICCV 2011, pp. 1623-1630, IEEE, Spain, 2011.

[19] E. A. L. Narváez and N. E. L. Narváez, "Point cloud denoising using robust principal component analysis," in Proceedings of the First International Conference on Computer Graphics Theory and Applications, Setúbal, Portugal, 2006.

[20] F. Zaman, Y. P. Wong, and B. Y. Ng, "Density-based denoising of point cloud," in Proceedings of the 9th International Conference on Robotic, Vision, Signal Processing and Power Applications, pp. 287-295, Springer, Singapore, 2017.

[21] R. B. Rusu and S. Cousins, "3D is here: Point Cloud Library (PCL)," in Proceedings of the IEEE International Conference on Robotics and Automation (ICRA '11), pp. 1-4, IEEE, Shanghai, China, 2011.

[22] I. Ashraf, S. Hur, and Y. Park, "An investigation of interpolation techniques to generate 2D intensity image from LIDAR data," IEEE Access, vol. 5, no. 99, pp. 8250-8260, 2017.

[23] M. F. Barnsley, "Fractal functions and interpolation," Constructive Approximation, vol. 2, no. 4, pp. 303-329, 1986.

[24] Y. M. Huang and C.-J. Chen, "3D Fractal reconstruction of terrain profile data based on digital elevation model," Chaos, Solitons \& Fractals, vol. 40, no. 4, pp. 1741-1749, 2009.

[25] K. Falconer, Fractal Geometry: Mathematical Foundations and Applications, K. Falconer, Ed., Wiley, 2nd edition, 2003.
[26] R. Małysz, "Convergence of trajectories in fractal interpolation of stochastic processes," Chaos Solitons \& Fractals, vol. 27, no. 5, pp. 1328-1338, 2006.

[27] C.-J. Chen, T.-Y. Lee, Y. M. Huang, and F.-J. Lai, "Extraction of characteristic points and its fractal reconstruction for terrain profile data," Chaos, Solitons \& Fractals, vol. 39, no. 4, pp. 1732$1743,2009$.

[28] J. Chen and T. Lee, "Fractal reality of random data compression for equal-interval series," Fractals-complex Geometry Patterns \& Scaling in Nature \& Society, vol. 8, no. 2, pp. 205-214, 2000.

[29] M. Barnsley, Fractals Everywhere, Academic Press, Boston, Mass, USA, 2nd edition, 1993.

[30] Z. Lanying, L. Zhixiong, and H. Zhanfeng, "Fractal parameters of the grade of road surface roughness," Computer and Communications, vol. 26, no. 6, pp. 158-161, 2008.

[31] C. Li, M. Liang, Y. Wang, and Y. Dong, "Vibration suppression using two terminal flywheel Part II: Application to vehicle passive suspension," Journal of Vibration and Control, vol. 18, no. 9, pp. 1353-1365, 2012.

[32] H. Pang, Y. Chen, J. Chen, and X. Liu, "Design of LQG controller for active suspension without considering road input signals," Shock and Vibration, vol. 2017, 13 pages, 2017.

[33] H. Habibullah, H. R. Pota, I. R. Petersen, and M. S. Rana, "Tracking of triangular reference signals using LQG controllers for lateral positioning of an AFM scanner stage," IEEE/ASME Transactions on Mechatronics, vol. 19, no. 4, pp. 1105-1114, 2014.

[34] K. S. Grewal, R. Dixon, and J. Pearson, "LQG controller design applied to a pneumatic stewart-gough platform," International Journal of Automation and Computing, vol. 9, no. 1, pp. 45-53, 2012.

[35] D. Miaomiao, Z. Dingxuan, B. Yang, and W. Lili, “Terminal sliding mode control for full vehicle active suspe-nsion systems," Journal of Mechanical Science and Technology, vol. 32, no. 6, pp. 2851-2866, 2018.

[36] A. Them, "The design of LQG controller for active suspension based on analytic hierarchy process," Mathematical Problems in Engineering, vol. 2010, 19 pages, 2010.

[37] T. L. Saaty, The Analytic Hierarchy Process, McGraw-Hill, New York, NY, USA, 1980

[38] B. Goenaga, L. Fuentes, and O. Mora, "Evaluation of the methodologies used to generate random pavement profiles based on the power spectral density: An approach based on the international roughness index," Ingenieria e Investigacion, vol. 37, no. 1, pp. 49-57, 2017.

[39] S. H. Yan and X. Tian, "Method of comparison matrix consistency adjustment based on AHP," Armament Automation, vol. 27, no. 4, article no. 14, pp. 8-9, 2008. 


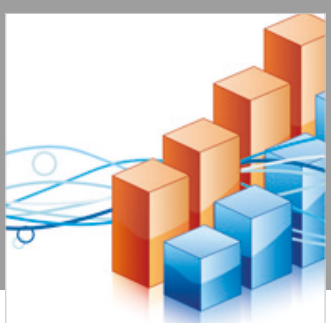

Advances in

Operations Research

\section{-n-m}
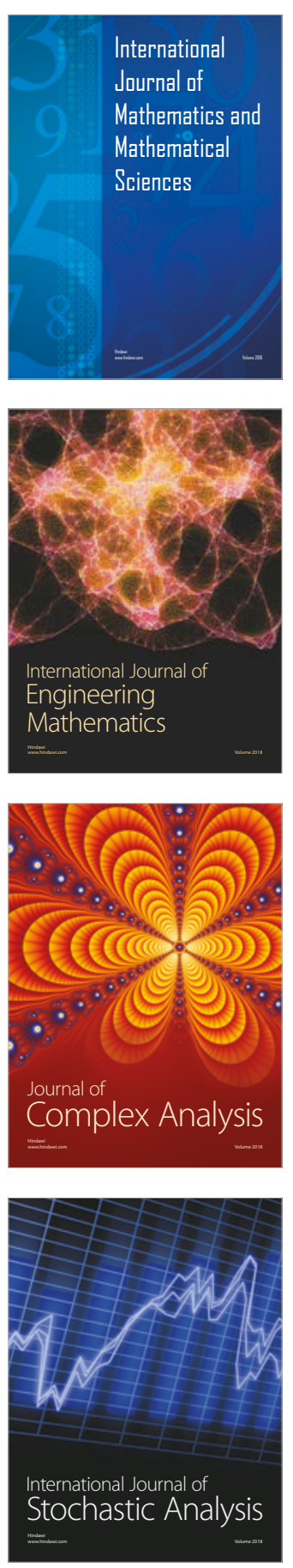
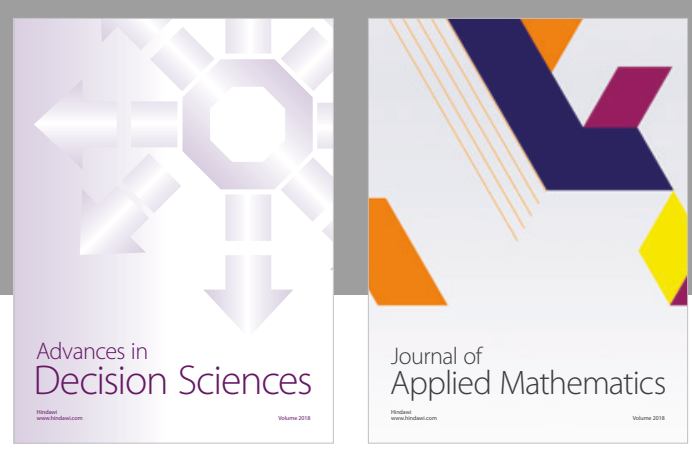

Journal of

Applied Mathematics
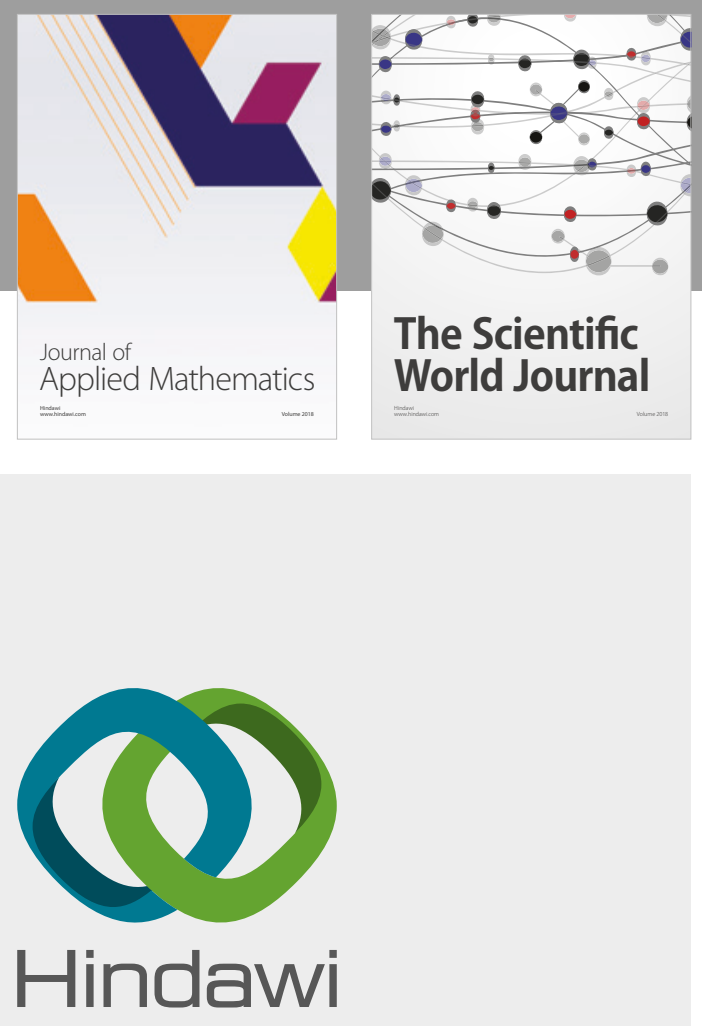

Submit your manuscripts at

www.hindawi.com

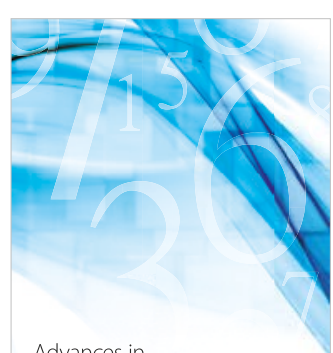

Advances in
Numerical Analysis
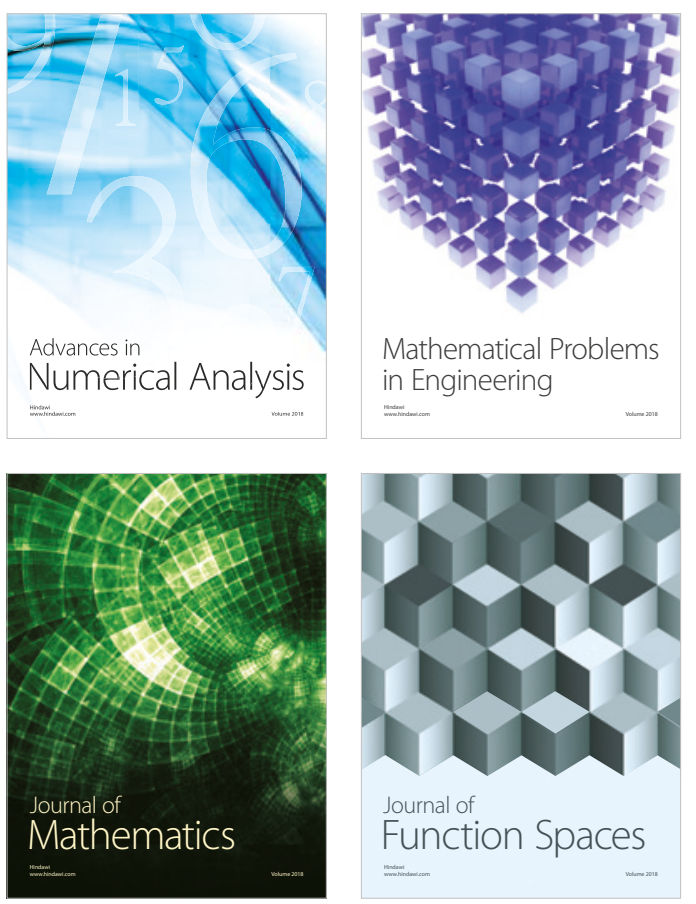

Mathematical Problems in Engineering

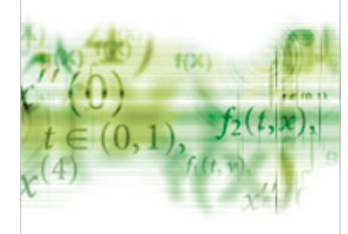

International Journal of

Differential Equations

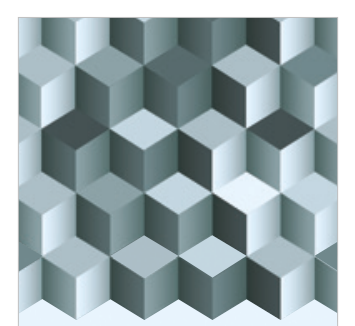

Journal of

Function Spaces
The Scientific

World Journal

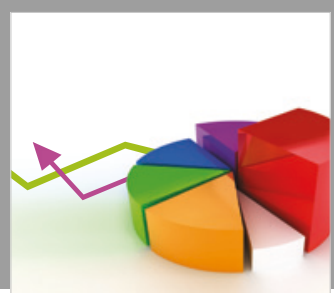

Journal of

Probability and Statistics
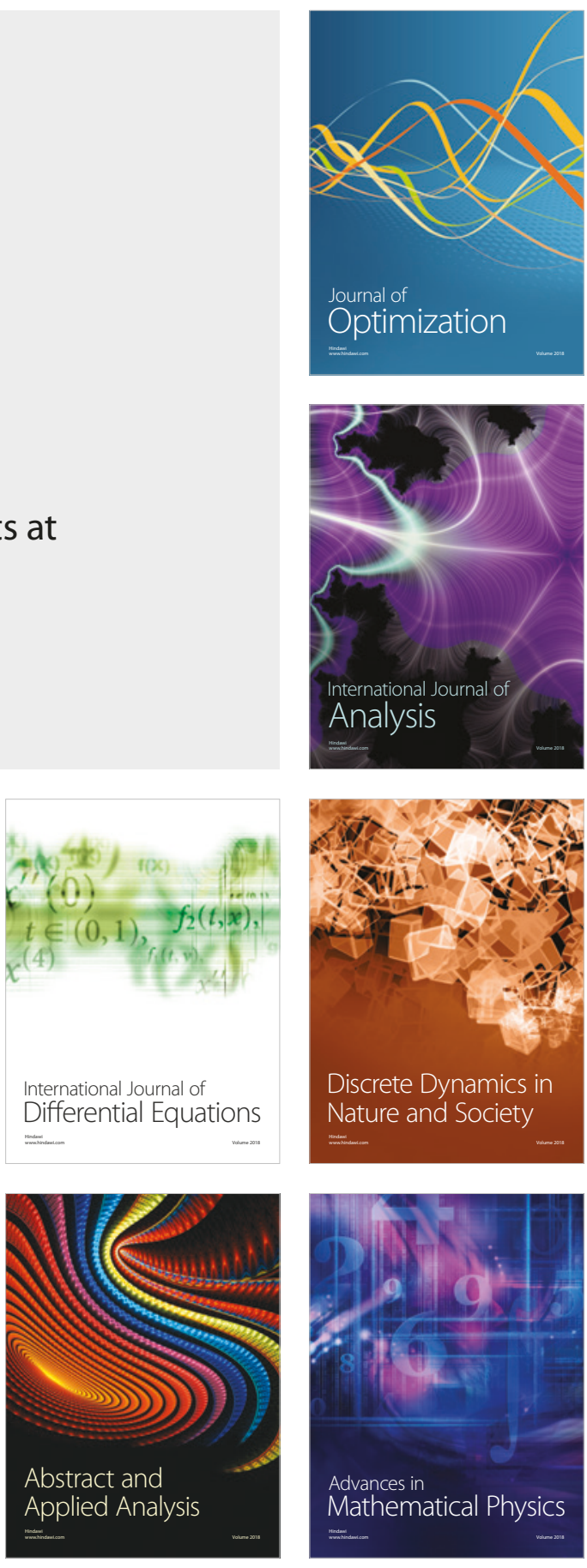